\title{
Holonomy of a class of bundles with fibre metrics
}

\author{
M. Crampin \\ Department of Mathematics, Ghent University \\ Krijgslaan 281, B-9000 Gent, Belgium \\ and D. J. Saunders \\ Department of Mathematics, Faculty of Science, \\ The University of Ostrava \\ 30. dubna 22, 70103 Ostrava, Czech Republic
}

May 28, 2010

\begin{abstract}
This paper is concerned with the holonomy of a class of spaces which includes Landsberg spaces of Finsler geometry. The methods used are those of Lie groupoids and algebroids as developed by Mackenzie. We prove a version of the Ambrose-Singer Theorem for such spaces. The paper ends with a discussion of how the results may be extended to Finsler spaces and homogeneous nonlinear connections in general.
\end{abstract}

MSC

53C60; 53C05, 53C29, 58H05.

\section{Keywords}

Fibre metric; Finsler space; holonomy algebroid; holonomy groupoid; horizontal distribution; Landsberg space; nonlinear connection.

\section{Introduction}

In most conventional accounts of the holonomy of a principal connection (the one by Kobayashi and Nomizu [5] has in our view rarely if ever been bettered, so we take it as a standard reference) attention is focussed on the holonomy group or algebra at a single point in the base manifold. It is, of course, proved that holonomy groups at different points are isomorphic, and 
likewise holonomy algebras: but these facts are treated almost in passing. Yet the holonomy objects are pointwise representatives of global structures, and one yearns for a theory of holonomy that adequately reflects this fact. It seems clear that, for example, the collection of holonomy algebras at all points of the base manifold $M$ will form a Lie algebra bundle over $M$ (a vector bundle whose fibres are Lie algebras, with local trivializations which are fibrewise Lie algebra isomorphisms with a standard Lie algebra fibre). One would like to know how the structure of this bundle is determined by the connection from which it is derived.

A theory of holonomy which provides the answers to such questions has been developed by Mackenzie [7]. Mackenzie's fundamental insight is that holonomy should be seen as a branch of the theory of Lie groupoids and Lie algebroids. Consider the case of a connection in a principal $G$-bundle $\pi: P \rightarrow M$. Parallel displacement along a piecewise smooth curve in $M$ joining points $x$ and $y$ is an isomorphism (strictly, $G$-equivariant diffeomorphism) of the fibre $P_{x}=\pi^{-1}(x)$ with the fibre $P_{y}$. The collection of all such fibre isomorphisms, for all curves $c$, is a groupoid $\Theta$ over $M$, with source projection the initial point $x$ of $c$, target projection the final point $y$. The holonomy group at a point $x$ is the vertex group at $x$ of $\Theta$, that is, the set of elements with the same source and target $x$. This groupoid is in fact a Lie groupoid, and has therefore associated with it a Lie algebroid $A \Theta$ over $M$, which is transitive; the holonomy Lie algebra bundle is just the kernel of $A \Theta$.

It seems to us that the theory of the holonomy of nonlinear connections, such as those that arise naturally in Finsler geometry, has so far lacked a really satisfactory conceptual framework, and we believe that the groupoid approach provides one. The underlying purpose of this paper is to initiate the development of the theory of the holonomy of nonlinear connections from this point of view. (For a survey of previous work on this subject see [6].) For technical reasons, which will be discussed in some detail at the end of the main part of the paper, we shall in fact restrict our attention for the most part to a certain class of geometrical structures admitting nonlinear connections, of which Landsberg spaces in Finsler geometry provide the best-known examples.

The fundamental tensor of a Finsler space over a manifold $M$ defines on each fibre $T_{x}^{\circ} M$ of the slit tangent bundle a Riemannian metric. This is evidently a particular case of a more general notion, that of a fibre bundle with a fibre metric. Quite a large proportion of this paper is devoted in fact to investigating relevant aspects of the differential geometry of such spaces in general, before we even consider such matters as nonlinear connections and 
holonomy. When we do come to deal with holonomy we restrict our attention to a special class of such spaces, which stand in relation to spaces with fibre metrics in general as Landsberg spaces do to the full class of Finsler spaces. For technical reasons we deal only with bundles with compact fibres (we can of course treat a Finsler structure as defined on a bundle having compact fibres by restricting to the indicatrix bundle). We prove a version of the Ambrose-Singer Theorem for this class of spaces, which specifies the holonomy Lie algebra bundle in terms of curvature. In any account of holonomy a distinction needs to be made between the full holonomy algebra at a point and the algebra generated by the covariant derivatives of the curvature; the latter is in general a proper subalgebra of the former, and indeed may differ from point to point. We discuss this matter in relation to Landsberg spaces in some detail.

The paper is laid out as follows. In the following section we give a brief resumé of Mackenzie's theory of holonomy. The geometry of bundles with fibre metrics is described in Section 3. The application to Landsberg spaces and their holonomy occupies Section 4 . We deal with covariant derivatives of the curvature in Section 5. Section 6 concludes the main part of the paper with a discussion of how our results might apply to Finsler spaces and nonlinear connections in greater generality. There are two appendices: in the first we give the basic definitions of groupoids and Lie algebroids, for the reader's convenience; in the second we present proofs of various results concerning vector bundles with connection which are needed in the main text.

\section{Groupoids and holonomy}

In this section we summarise some general results regarding groupoids and holonomy. The reader is referred to [7, Section 6.3] for details of the proofs. We start by describing a connection on a locally trivial Lie groupoid $\Omega$ over a connected base manifold $M$, with source projection $\alpha$ and target projection $\beta$, as a means of lifting curves in $M$ to curves in $\Omega$.

Definition. Let $\mathcal{C}(M)$ denote the set of continuous, piecewise-smooth curves $c:[0,1] \rightarrow M$, and let $\mathcal{C}(\Omega)$ denote the corresponding set of curves in $\Omega$. A connection on $\Omega$ is a map $\Gamma: \mathcal{C}(M) \rightarrow \mathcal{C}(\Omega), c \mapsto c^{\Gamma}$, satisfying the following properties:

$$
\begin{aligned}
& \text { 1. } c^{\Gamma}(0)=1_{c(0)} \text { and, for all } t \in[0,1], \\
& \qquad \alpha\left(c^{\Gamma}(t)\right)=c(0), \quad \beta\left(c^{\Gamma}(t)\right)=c(t) ;
\end{aligned}
$$


2. if $[a, b] \subset[0,1]$, and if $\phi:[0,1] \rightarrow[a, b]$ is a diffeomorphism, then

$$
(c \circ \phi)^{\Gamma}=r_{c^{\Gamma}(\phi(0))^{-1}} \circ c^{\Gamma} \circ \phi ;
$$

3. if $c$ is smooth at $t \in[0,1]$ then so is $c^{\Gamma}$;

4. if $c_{1}, c_{2} \in \mathcal{C}(M)$ and, for some $t \in[0,1]$,

$$
\dot{c}_{1}(t)=\dot{c}_{2}(t)
$$

then

$$
\dot{c}_{1}^{\Gamma}(t)=\dot{c}_{2}^{\Gamma}(t)
$$

5. if $c_{1}, c_{2}, c_{3} \in \mathcal{C}(M)$ and, for some $t \in[0,1]$,

$$
\dot{c}_{1}(t)+\dot{c}_{2}(t)=\dot{c}_{3}(t)
$$

then

$$
\dot{c}_{1}^{\Gamma}(t)+\dot{c}_{2}^{\Gamma}(t)=\dot{c}_{3}^{\Gamma}(t) .
$$

A consequence of the properties above is that lifts are consistent with reparametrization, so that the definition of a lift may be extended to curves whose domains are arbitrary intervals in $\mathbb{R}$.

In the remainder of this paper we shall be concerned with Lie algebroids as much as with Lie groupoids, and so we shall also need the equivalent definition, that of an infinitesimal connection.

Definition. Let $\pi: A \rightarrow M$ be a transitive Lie algebroid, with anchor map $a: A \rightarrow T M$. A connection on $A$ is a vector bundle map $\gamma: T M \rightarrow A$ over the identity on $M$ satisfying $a \circ \gamma=\operatorname{id}_{T M}$. If $\Omega$ is a locally trivial Lie groupoid then an infinitesimal connection on $\Omega$ is a connection on the Lie algebroid $A \Omega$.

Proposition 1. There is a bijective correspondence between connections $\Gamma$ and infinitesimal connections $\gamma$ on $\Omega$, given by

$$
\dot{c}^{\Gamma}(t)=r_{c^{\Gamma}(t) *}(\gamma(\dot{c}(t))) .
$$

We now consider a fixed connection $\Gamma$ on $\Omega$. It is a consequence of the definition that the lift of a constant curve is an identity in $\Omega$, that the lift of a concatenation of curves is the product of the separate lifts, and that the lift of a curve traversed in the reverse direction is the inverse of the lift of the original curve. We may therefore make the following definition. 
Definition. The holonomy subgroupoid $\Theta \subset \Omega$ of the connection $\Gamma$ is defined by

$$
\Theta=\left\{c^{\Gamma}(1): c \in \mathcal{C}(M)\right\}
$$

For any $x \in M$ the holonomy group of $\Gamma$ at $x$ is defined by

$$
H_{x}=\left\{c^{\Gamma}(1) \in \Theta: c(0)=c(1)=x\right\},
$$

and the restricted holonomy group is the normal subgroup $H_{x}^{\circ} \triangleleft H_{x}$ where the loops $c$ are contractible in $M$.

Theorem 1. The holonomy groupoid $\Theta$ is a Lie subgroupoid of $\Omega$.

Proof. It is clear that $\Theta$ will be a subgroupoid under the operations induced from $\Omega$. The proof that it is a Lie subgroupoid is a modification of the classical proof that the holonomy groups of a connection on a principal bundle are Lie groups, as described in [5, Theorem 4.2].

The proof starts by writing $\Omega_{x, x}$ for the subset $\{\omega \in \Omega: \alpha(\omega)=\beta(\omega)=$ $x\}$; it is clear that $\Omega_{x, x}$ is both a group and a submanifold of $\Omega$; the Lie groupoid properties of $\Omega$ then imply that $\Omega_{x, x}$ is a Lie group. Thus $H_{x}$ and $H_{x}^{\circ}$ are (topological) subgroups of $\Omega_{x, x}$.

The next step of the argument is to show that $H_{x}^{\circ}$ is path-connected. An intricate argument known as the Factorization Lemma (see [5, Appendix 7]) shows that it is sufficient to consider elements $c^{\Gamma}(1) \in H_{x}^{\circ}$ of a particular type, where the curve $c$ is known as a lasso, and is a small loop based at some point $y \in M$ preceded by a path from $x$ to $y$ and followed by the reverse path from $y$ to $x$. The small loop may be taken to lie within a single coordinate chart of $M$, and thus the lasso $c$ may be shrunk to the single point $x \in M$, giving a path in $H_{x}^{\circ}$ from $c^{\Gamma}(1)$ to the identity. It then follows from a standard theorem that the restricted holonomy group $H_{x}^{\circ}$, as a path-connected subgroup of a Lie group, is itself a Lie group.

It follows from this that the full holonomy group $H_{x}$ is also a Lie group. Here we use the fact that $M$ is second countable, a consequence of the assumed connectedness of $M$ and the standard assumption that $M$ is paracompact. Thus the homotopy group $\pi_{1}(M, x)$ is countable, and the existence of a homomorphism $\pi_{1}(M, x) \rightarrow H_{x} / H_{x}^{\circ}$ shows that the quotient group $H_{x} / H_{x}^{\circ}$ is countable. It then follows from another standard theorem that $H_{x}$ is a Lie group.

Finally, we have to show that $\Theta$ is a Lie groupoid. The argument here is similar to the one we give below in Theorem 3, using the Lie group structure of each $H_{x}$ and the manifold structure of $M$; more details may be found in [7, Theorem 6.3.19]. 
Given the connection $\Gamma$ on $\Omega$, the corresponding infinitesimal connection $\gamma$ will be defined on the Lie algebroid $A \Omega$. We have seen that the holonomy groupoid $\Theta$ of $\Gamma$ is a Lie groupoid, and so it will have a Lie algebroid $A \Theta$ which may be identified with a Lie subalgebroid of $A \Omega$. We now wish to see if this particular subalgebroid may be characterised in some way by $\gamma$. To find such a characterisation, we need to use the covariant differentiation and the curvature associated with $\gamma$.

Definition. Let $\gamma$ be an infinitesimal connection on $A \Omega$. Define, for any vector field $X \in \mathfrak{X}(M)$, the covariant derivative map $\nabla_{X}^{\gamma}: \operatorname{Sec}(A \Omega) \rightarrow$ $\operatorname{Sec}(A \Omega)$ by

$$
\nabla_{X}^{\gamma}(\phi)=[\gamma(X), \phi] .
$$

Define the curvature of the connection to be the map $R^{\gamma}: \mathfrak{X}(M) \times \mathfrak{X}(M) \rightarrow$ $\operatorname{Sec}(A \Omega)$ given by

$$
R^{\gamma}(X, Y)=\gamma([X, Y])-[\gamma(X), \gamma(Y)]
$$

describing the extent to which $\gamma: \mathfrak{X}(M) \rightarrow \operatorname{Sec}(A \Omega)$ fails to be a Lie algebra homomorphism.

We shall also need to consider Lie algebra sub-bundles $L$ of $L \Omega$, the kernel of $A \Omega$. Given such an $L$, define $A \subset A \Omega$ as the span of certain sections of $A \Omega$,

$$
\operatorname{Sec}(A)=\{\phi \in \operatorname{Sec}(A \Omega): \phi-\gamma a(\phi) \in \operatorname{Sec}(L)\} .
$$

Proposition 2. If $L$ is invariant under covariant differentiation, so that $\nabla_{X}^{\gamma}(\operatorname{Sec}(L)) \subset \operatorname{Sec}(L)$ for every $X \in \mathfrak{X}(M)$, and if the curvature takes its values in $L$, so that $R^{\gamma}(X, Y) \in \operatorname{Sec}(L)$ for every $X, Y \in \mathfrak{X}(M)$, then $A \rightarrow M$ is a Lie algebroid with kernel $L$.

The fundamental result ([7, Theorem 6.4.20]) is as follows.

Theorem 2. There is a least Lie algebra sub-bundle $(L \Omega)^{\gamma}$ of $L \Omega$ satisfying the conditions of the proposition above, and the corresponding Lie subalgebroid $(A \Omega)^{\gamma}$ of $A \Omega$ satisfies

$$
(A \Omega)^{\gamma}=A \Theta .
$$

This description may appear somewhat formal, so let us explain how it works in a familiar situation, that of a linear connection on a manifold $M$. We may think of such a connection in various ways: as a covariant derivative; 
as a law of parallel transport; or as a horizontal distribution on the tangent bundle $T M$, spanned by local vector fields

$$
\left(\frac{\partial}{\partial x^{i}}\right)^{\mathrm{H}}=\frac{\partial}{\partial x^{i}}-\Gamma_{i k}^{j} u^{k} \frac{\partial}{\partial u^{j}}
$$

in the usual notation.

We first remark that if a vector field on $T M$ takes the form

$$
\xi^{i} \frac{\partial}{\partial x^{i}}+\eta_{k}^{j} u^{k} \frac{\partial}{\partial u^{j}}
$$

in terms of some coordinates $x^{i}$ on $M$, where $\xi^{i}$ and $\eta_{k}^{j}$ are functions of the $x^{i}$ alone, then (as may easily be checked) it takes the same form when new coordinates are chosen for $M$. We call such a vector field projectable fibre-linear; evidently it projects onto $\xi^{i} \partial / \partial x^{i}$ on $M$.

To start the groupoid description of the holonomy of a linear connection we take for the ambient groupoid $\Omega$ the Lie groupoid whose elements with source $x$ and target $y$ are the (linear) fibre isomorphisms $T_{x} M \rightarrow T_{y} M$. The elements of the Lie algebroid $A \Omega$ over $x$ are projectable fibre-linear vector fields along $T_{x} M \rightarrow T M$ : such a vector field is defined on, but is not necessarily tangent to, the fibre $T_{x} M$, and takes the form displayed above, where now $\xi^{i}$ and $\eta_{k}^{j}$ are constants. Notice that points of $(A \Omega)_{x}$ are vector fields along $T_{x} M \rightarrow T M$. The space of projectable fibre-linear vector fields along $T_{x} M \rightarrow T M$ is finite-dimensional, and $A \Omega$ is indeed a vector bundle. Sections of $A \Omega \rightarrow M$ are projectable fibre-linear vector fields on $T M$, and the Lie algebroid bracket is just the ordinary bracket of vector fields on $T M$ (of course the bracket of projectable fibre-linear vector fields is projectable fibre-linear). The anchor is projection, and the kernel $L \Omega$ consists of vertical fibre-linear vector fields on $T M$.

We turn next to the connection on $\Omega$ corresponding to the given linear connection. Let $c \in \mathcal{C}(M)$ : we have to define $c^{\Gamma} \in \mathcal{C}(\Omega)$. Thus for each $t \in[0,1], c^{\Gamma}(t)$ should be a fibre isomorphism $T_{c(0)} M \rightarrow T_{c(t)} M$. Parallel translation along $c$ from $c(0)$ to $c(t)$ is such a fibre isomorphism, and if we take this for $c^{\Gamma}(t)$ we see it satisfies all the requirements for a connection on $\Omega$. The corresponding element of the holonomy groupoid is parallel translation along $c$ from $c(0)$ to $c(1)$.

The corresponding infinitesimal connection $\gamma: T M \rightarrow A \Omega$ is just the horizontal lift: for $v \in T_{x} M, v=v^{i} \partial / \partial x^{i}$

$$
\gamma(v)=v^{\mathrm{H}}=v^{i}\left(\frac{\partial}{\partial x^{i}}-\Gamma_{i k}^{j} u^{k} \frac{\partial}{\partial u^{j}}\right) .
$$


Of course we have to ensure that $\gamma(v)$ is an element of $(A \Omega)_{x}$, that is, a projectable fibre-linear vector field along $T_{x} M \rightarrow T M-$ which indeed $v^{\mathrm{H}}$ is.

Now any projectable fibre-linear vector field on $T M$ (section of $A \Omega \rightarrow M$ in other words) can be written uniquely in the form $X^{\mathrm{H}}+S^{\mathrm{V}}$ where $X$ is a vector field and $S$ a type $(1,1)$ tensor field on $M$, and the 'vertical lift' $S^{\mathrm{v}}$ of $S$ is given by

$$
S^{\mathrm{V}}=S_{j}^{i} u^{j} \frac{\partial}{\partial u^{i}} .
$$

(One needs the connection to make the vertical part a tensor: $\eta_{j}^{i}$ in the previous incarnation is not a tensor.) We can therefore identify $A \Omega$ with $T M \oplus T_{1}^{1} M$. By straightforward calculations

$$
\begin{aligned}
{\left[X^{\mathrm{H}}, Y^{\mathrm{H}}\right] } & =[X, Y]^{\mathrm{H}}-R(X, Y)^{\mathrm{V}} ; \\
{\left[X^{\mathrm{H}}, T^{\mathrm{V}}\right] } & =\left(\nabla_{X} T\right)^{\mathrm{V}} ; \\
{\left[S^{\mathrm{V}}, T^{\mathrm{V}}\right] } & =-\{S, T\}^{\mathrm{V}},
\end{aligned}
$$

where $R$ is the curvature tensor of the linear connection (so $R(X, Y)$ is a type $(1,1)$ tensor), and $\{S, T\}$ is the commutator of $S$ and $T$ (considered as matrices). So the bracket of sections of $A \Omega$ is given by

$$
[X \oplus S, Y \oplus T]=[X, Y] \oplus\left(-R(X, Y)+\nabla_{X} T-\nabla_{Y} S-\{S, T\}\right) .
$$

In particular, we can identify $L \Omega$ with $T_{1}^{1} M$, with bracket the negative of the commutator. The objects of interest now are the Lie algebra sub-bundles of $T_{1}^{1} M$ whose section spaces are closed under covariant derivative and contain all curvature tensors $R(X, Y)$; the holonomy Lie algebra bundle is the least such.

\section{$3 \quad$ Fibre metrics}

We now turn to the definition and properties of fibre metrics: but first we prove a result which has an important role to play at several points in this section.

Proposition 3. Let $\pi: E \rightarrow M$ be a fibre bundle whose standard fibre is compact. Let $Z$ be any vector field on $E$, with flow $\chi_{t}$. Then for any $x \in M$ there is an open interval I containing 0 such that for all $u \in E_{x}, \chi_{t}(u)$ is defined for all $t \in I$. If $Z$ is projectable to a vector field $\bar{Z} \in \mathfrak{X}(M)$, its flow $\chi_{t}$ is projectable to the flow $\bar{\chi}_{t}$ of $\bar{Z}$; then $\bar{\chi}_{t}(x)$ is also defined for all $t \in I$. 
Proof. For each $u \in E_{x}$ there is an open neighbourhood $U_{u}$ of $u$ in $E$ and an open interval $I_{u}$ containing 0 such that $\chi_{t}(v)$ is defined for all $t \in I_{u}$ and $v \in U_{u}$, and in particular for all $v \in U_{u} \cap E_{x}$. The open sets $U_{u} \cap E_{x}$ cover $E_{x}$, so we can find a finite subcover: let $I$ be the intersection of the corresponding open intervals $I_{u}$. Then $I$, being the intersection of a finite number of open intervals, is open, and contains 0 , and for every $v \in E_{x}$, $\chi_{t}(v)$ is defined for all $t \in I$.

Let $\pi: E \rightarrow M$ be a fibre bundle, and $V \pi \rightarrow E$ the vertical sub-bundle of $T E$. By a type $(0,2)$ fibre tensor on $E$ we mean a smooth bilinear map $V \pi \times{ }_{E} V \pi \rightarrow \mathbb{R}$. If $g$ is a type $(0,2)$ fibre tensor on $E$ then $g_{x}$, its restriction to $E_{x}=\pi^{-1}(x)$, is a type $(0,2)$ tensor field on $E_{x}$. A fibre metric on $E$ is a type $(0,2)$ fibre tensor for which $g_{x}$ is a Riemannian metric on $E_{x}$ for all $x$.

Let $Z$ be a projectable vector field on $E$; then for any vector field $V$ on $E$ which is vertical over $M,[Z, V]$ is vertical. So for any vertical vector fields $V, W$ on $E$ we may set

$$
\mathcal{L}_{Z} g(V, W)=Z(g(V, W))-g([Z, V], W)-g(V,[Z, W]) .
$$

Clearly, $\mathcal{L}_{Z} g(V, W)$ is $C^{\infty}(E)$-bilinear in $V$ and $W$, so this formula defines a type $(0,2)$ fibre tensor $\mathcal{L}_{Z} g$, which is evidently symmetric.

The operator $\mathcal{L}_{Z}$ is a form of Lie derivative, as can be seen in two different ways. In the first place, we can think of $V \mapsto[Z, V]$ as a Lie derivative of vertical vector fields (identifying the Lie derivative and Lie bracket in the usual way); then the formula for $\mathcal{L}_{Z} g$ above can be written

$$
\mathcal{L}_{Z} g(V, W)=\mathcal{L}_{Z}(g(V, W))-g\left(\mathcal{L}_{Z} V, W\right)-g\left(V, \mathcal{L}_{Z} W\right),
$$

which mimics the usual method of extending the Lie derivative from vector fields to tensor fields. This formulation makes it clear that to evaluate $\mathcal{L}_{Z} g(V, W)$ at any $u \in E$ we need consider only the values of $V$ and $W$ (and of course $g$ ) along the integral curve of $Z$ through $u$.

Secondly, when the fibres of $E$ are compact we can interpret the formula in terms of flows, as follows. Since $Z$ is projectable, to $\bar{Z} \in \mathfrak{X}(M)$, its flow $\chi_{t}$ is projectable to the flow $\bar{\chi}_{t}$ of $\bar{Z}$. Given $x \in M$, by Proposition $3 \chi_{t}(u)$ is defined for all $u \in E_{x}$ for $t$ in some open interval $I$ containing 0 . Denote the restriction of $\chi_{t}, t \in I$, to $E_{x}$ by $\chi_{x ; t}$; thus $\chi_{x ; t}$ is a diffeomorphism of $E_{x}$ with $E_{\bar{\chi}_{t}(x)}$. The pullback $\chi_{x ; t}^{*}\left(g_{\bar{\chi}_{t}(x)}\right)$ is defined for any $t \in I$, and is another symmetric type $(0,2)$ tensor field on $E_{x}$. We claim that

$$
\left.\frac{d}{d t} \chi_{x ; t}^{*}\left(g_{\bar{\chi}_{t}(x)}\right)\right|_{t=0}=\left(\mathcal{L}_{Z} g\right)_{x} .
$$


To establish the claim, choose vertical vector fields $V_{x}, W_{x}$ on $E_{x}$, and extend them along $x_{t}=\bar{\chi}_{t}(x)$ by Lie transport, so that $V_{x_{t}}=\chi_{x ; t *} V_{x}$. Then

$$
\left(\chi_{x ; t}^{*} g_{x_{t}}\right)\left(V_{x}, W_{x}\right)=g_{x_{t}}\left(\chi_{x ; t *} V_{x}, \chi_{x ; t *} W_{x}\right)=g_{x_{t}}\left(V_{x_{t}}, W_{x_{t}}\right)=g(V, W)\left(x_{t}\right) .
$$

Thus

$$
\left.\frac{d}{d t} \chi_{x ; t}^{*} g_{x_{t}}\right|_{t=0}\left(V_{x}, W_{x}\right)=Z_{x}(g(V, W)) .
$$

But $\mathcal{L}_{Z} V=\mathcal{L}_{Z} W=0$ by construction, so in this case

$$
\left.\frac{d}{d t} \chi_{x ; t}^{*} g_{x_{t}}\right|_{t=0}\left(V_{x}, W_{x}\right)=\left(\mathcal{L}_{Z} g\right)_{x}\left(V_{x}, W_{x}\right) .
$$

But each of $d / d t\left(\chi_{x ; t}^{*} g_{x_{t}}\right)_{t=0}$ and $\left(\mathcal{L}_{Z} g\right)_{x}$ is a tensor field on $E_{x}$, and so they are equal (as tensors).

Of course if $Z_{1}$ and $Z_{2}$ are projectable so is $\left[Z_{1}, Z_{2}\right]$. It is easy to see that

$$
\mathcal{L}_{Z_{1}}\left(\mathcal{L}_{Z_{2}} g\right)-\mathcal{L}_{Z_{2}}\left(\mathcal{L}_{Z_{1}} g\right)=\mathcal{L}_{\left[Z_{1}, Z_{2}\right]} g
$$

So far, so fairly predictable; but now an unexpected feature emerges. For any $f \in C^{\infty}(M)$,

$\mathcal{L}_{f Z} g(V, W)=f Z(g(V, W))-g([f Z, V], W)-g(V,[f Z, W])=f \mathcal{L}_{Z} g(V, W)$,

since $V f=W f=0$. That is to say, $\mathcal{L}_{Z} g(V, W)$ is $C^{\infty}(M)$-linear in $Z$. Thus so far as its dependence on $Z$ is concerned, $\mathcal{L}_{Z} g$ behaves like a covariant derivative rather than a Lie derivative. In particular, $\left(\mathcal{L}_{Z} g\right)_{x}$ depends only on the value of $Z$ on $E_{x}$. To put things another way, for any vector field $Z_{x}$ along $E_{x} \rightarrow E$ which is projectable to $T_{x} M$, there is a well-defined symmetric type $(0,2)$ tensor field $\mathcal{L}_{Z_{x}} g$ on $E_{x}$, given by $\mathcal{L}_{Z_{x}} g=\left(\mathcal{L}_{Z} g\right)_{x}$ where $Z$ is any vector field on $E$ defined in a neighbourhood of $E_{x}$ which agrees with $Z_{x}$ on $E_{x}$. This feature is clear also from the coordinate representation of $\mathcal{L}_{Z} g$. Take coordinates $x^{i}$ on $M$ and $u^{a}$ on the fibre; let

$$
Z=\xi^{i} \frac{\partial}{\partial x^{i}}+\eta^{a} \frac{\partial}{\partial u^{a}}, \quad g\left(\frac{\partial}{\partial u^{a}}, \frac{\partial}{\partial u^{b}}\right)=g_{a b},
$$

where $\xi^{i}=\xi^{i}(x)$; then

$$
\left(\mathcal{L}_{Z} g\right)_{a b}=\xi^{i} \frac{\partial g_{a b}}{\partial x^{i}}+\eta^{c} \frac{\partial g_{a b}}{\partial u^{c}}+g_{c b} \frac{\partial \eta^{c}}{\partial u^{a}}+g_{a c} \frac{\partial \eta^{c}}{\partial u^{b}} .
$$

The point to note is that no derivatives of the components of $Z$ with respect to the $x^{i}$ appear on the right-hand side. It is also clear from this formula, if 
it wasn't already, that if $Z_{x}$ is actually vertical then $\mathcal{L}_{Z_{x}} g$ is just the ordinary Lie derivative of $g_{x}$ considered as a tensor field on $E_{x}$.

We call $\mathcal{L}_{Z} g=0$ the isometry equation for (projectable) vector fields, and $\mathcal{L}_{Z_{x}} g=0$ the isometry equation at $x$. Every vector field solution of the isometry equation gives rise to a solution of the isometry equation at $x$, but it is not necessarily the case that a solution of the isometry equation at $x$ can be extended to a vector field solution, even locally.

Suppose that $Z$ is a solution of the isometry equation; denote its flow by $\chi_{t}$, and the flow of its projection $\bar{Z}$ by $\bar{\chi}_{t}$. We know from Proposition 3 that for any $x \in M, \chi_{x ; t}$ is well-defined for all $t$ in some open interval $I$ containing 0 . It follows from the fact that $\mathcal{L}_{Z} g=0$, by a standard argument, that

$$
\frac{d}{d t}\left(\chi_{x ; t}^{*} g_{x_{t}}\right)=0
$$

for all $t \in I$, and hence that $\chi_{x ; t}^{*} g_{x_{t}}=g_{x}$. Thus a solution of the isometry equation for vector fields is the infinitesimal generator of fibre isometries of the fibre metric. We call such a vector field an infinitesimal fibre isometry of the fibre metric, and denote the space of infinitesimal fibre isometries by $\mathfrak{J}$. Then $\mathfrak{J}$ is a $C^{\infty}(M)$-module of vector fields on $E$ which is closed under bracket.

The solutions of the isometry equation at $x$ form a vector space over $\mathbb{R}$ which we denote by $\mathfrak{J}^{x}$. Those solutions which are vertical are simply infinitesimal isometries, or Killing fields, of the metric $g_{x}$. Since a solution of the isometry equation at $x$ is projectable to $T_{x} M$, the linear map $\pi_{*}$ restricts to a linear map $\mathfrak{J}^{x} \rightarrow T_{x} M$, and we see that the solution space $\mathfrak{J}^{x}$ is of finite dimension at most $m+\frac{1}{2} n(n-1)$ where $m=\operatorname{dim} M$ and $n=\operatorname{dim} E_{x}$. The kernel of $\left.\pi_{*}\right|_{\mathfrak{J}^{x}}$, which we denote by $\mathfrak{K}^{x}$, is the space of infinitesimal isometries of $g_{x}$, and in particular is a Lie algebra (under Lie bracket), not just a vector space.

It is tempting to think of $\mathfrak{J}$ as consisting of the sections of a vector bundle over $M$ whose fibre at $x$ is $\mathfrak{J}^{x}$, but this will not normally be permissible: for example, there is no guarantee that the spaces $\mathfrak{J}^{x}$ at different points $x$ are isomorphic. We next describe a situation in which this difficulty does not arise.

Consider a fibre bundle $\pi: E \rightarrow M$ whose standard fibre is compact and whose base is connected, which is equipped with a fibre metric $g$ such that the map $\pi_{*}: \mathfrak{J} \rightarrow \mathfrak{X}(M)$, taking each infinitesimal fibre isometry to its projection on $M$, is surjective. In such a case we say that $\mathfrak{J}$ is transitive.

Proposition 4. Let $\pi: E \rightarrow M$ be a fibre bundle with compact standard fibre 
and connected base, equipped with a fibre metric $g$ such that $\mathfrak{J}$ is transitive. The fibres of E, considered as Riemannian manifolds, are pairwise isometric.

Proof. Let $x$ and $y$ be points of $M$ that both lie on an integral curve of some vector field $X$. We show that $E_{x}$ and $E_{y}$ are isometric. Let $\varphi_{t}$ be the flow of $X$, and suppose that $y=\varphi_{s}(x)$ (without loss of generality we may assume that $s>0)$. By assumption there is a vector field $\tilde{X}$ on $E$ such that $\tilde{X} \in \mathfrak{J}$ and $\pi_{*} \tilde{X}=X$. Let $\tilde{\varphi}_{t}$ be the flow of $\tilde{X}$ : then $\pi \circ \tilde{\varphi}_{t}=\varphi_{t}$, and for any $z \in M$ there is an open interval $I$ containing 0 such that $\tilde{\varphi}_{t}$ is an isometry of $E_{z}$ with $E_{\varphi_{t}(z)}$ for all $t \in I$. This holds in particular for $z=\varphi_{r}(x)$ for all $r \in[0, s]$. So we have a covering of $[0, s]$ by open intervals say $\left(r-\delta_{r}, r+\delta_{r}\right)$ on each of which $\tilde{\varphi}_{t}\left(E_{\varphi_{r}(x)}\right)$ is defined. From this covering we can extract a finite subcovering. Then using the one-parameter group property we see that in fact $\tilde{\varphi}_{t}\left(E_{x}\right)$ is defined for all $t \in[0, s]$, and therefore $\tilde{\varphi}_{s}: E_{x} \rightarrow E_{y}$ is an isometry (and indeed $\tilde{\varphi}_{r}: E_{x} \rightarrow E_{\varphi_{r}(x)}$ is an isometry for all $r \in[0, s]$ ). Now any pair of points in $M$ can be joined by a piecewise smooth curve which is made up of segments each of which is part of the integral curve of some vector field; the general result follows.

Since the fibres are all isometric, we can choose a Riemannian manifold isometric to each of them, for example any one fibre $E_{x}$ with its metric $g_{x}$. We denote this representative Riemannian manifold by $\mathcal{E}$.

Proposition 5. Let $\pi: E \rightarrow M$ be a fibre bundle with compact standard fibre and connected base, equipped with a fibre metric $g$ such that $\mathfrak{J}$ is transitive. About every point of $M$ there is a neighbourhood $U$ and a local trivialization $\tau: U \times\left.\mathcal{E} \rightarrow E\right|_{U}$, compatible with the smooth bundle structure, such that for all $x \in U$, the map $\tau_{x}: \mathcal{E} \rightarrow E_{x}$ given by $\tau_{x}=\tau(x, \cdot)$ is an isometry.

Proof. Fix a point in $M$ and take a coordinate neighbourhood $U$ with this point as origin; we shall denote it by $o$. Without loss of generality we may assume that the image of $U$ is the open unit ball in $\mathbb{R}^{m}$, and we may further assume that $U$ is contained in some neighbourhood over which $E$ is locally trivial. Since $\mathfrak{J}$ is a $C^{\infty}(M)$-module, with each coordinate field $X_{i}=\partial / \partial x^{i}$ we may associate a vector field $\tilde{X}_{i}$ on $E$ such that $\tilde{X}_{i} \in \mathfrak{J}$ and $\pi_{*} \tilde{X}_{i}=X_{i}$ on $U$. For each $x \in U$ let $r_{x}$ be the ray joining the origin to $x$ with respect to the coordinates: it is an integral curve of $x^{i}(x) X_{i}$, where the $x^{i}(x)$ are the coordinates of $x$ (which of course are to be treated as constants). Since $\mathfrak{J}$ is a vector space over $\mathbb{R}, x^{i}(x) \tilde{X}_{i} \in \mathfrak{J}$. Let $\sigma_{x}: E_{o} \rightarrow E_{x}$ be the isometry determined by $x^{i}(x) \tilde{X}_{i}$. Let $u^{a}$ be fibre coordinates on $E$ over $U$. Then

$$
\tilde{X}_{i}=\frac{\partial}{\partial x^{i}}+N_{i}^{a} \frac{\partial}{\partial u^{a}}
$$


for certain functions $N_{i}^{a}$ on $\left.E\right|_{U}$, and so

$$
x^{i}(x) \tilde{X}_{i}=x^{i}(x)\left(\frac{\partial}{\partial x^{i}}+N_{i}^{a} \frac{\partial}{\partial u^{a}}\right) .
$$

Thus for any $u \in E_{o}, u=\left(0, u^{a}\right)$, the fibre coordinates of $\sigma_{x}(u)$ are determined as the solution of the system of ordinary differential equations

$$
\dot{u}^{a}=x^{i}(x) N_{i}^{a}\left(t x^{j}(x), u^{b}\right), \quad u^{a}(0)=u^{a},
$$

in which the coordinates $x^{i}(x)$ play the role of parameters. But the solutions of such equations depend smoothly on parameters, so $\sigma_{x}(u)$ depends smoothly on the coordinates $\left(x^{i}, u^{a}\right)$. Now let $\phi: \mathcal{E} \rightarrow E_{o}$ be an isometry. We define $\tau: U \times\left.\mathcal{E} \rightarrow E\right|_{U}$ by $\tau(x, v)=\sigma_{x}(\phi(v))$ for $v \in \mathcal{E}$. Then $\tau$ is a local trivialization, compatible with the smooth bundle structure, such that for all $x \in U, \tau_{x}=\sigma_{x} \circ \phi: \mathcal{E} \rightarrow E_{x}$ is an isometry.

For any bundle $E \rightarrow M$ with fibre metric $g$ the set of isometries between fibres is evidently a groupoid, which we call the fibre-isometry groupoid (the precise definition is the first paragraph of the proof of the following theorem).

Theorem 3. The fibre-isometry groupoid of a fibre bundle $\pi: E \rightarrow M$ with compact standard fibre and connected base, equipped with a fibre metric $g$ such that $\mathfrak{J}$ is transitive, is a locally trivial Lie groupoid. The corresponding Lie algebroid has the spaces $\mathfrak{J}^{x}$ for its fibres and the space $\mathfrak{J}$ as its sections.

Proof. Given the bundle $\pi: E \rightarrow M$, we let $\Omega_{y, x}$ denote the set of isometries from the fibre $E_{x}$ to the fibre $E_{y}$, and let $\Omega=\bigcup_{x, y \in M} \Omega_{y, x}$. If $\theta \in \Omega_{y, x}$ put $\alpha(\theta)=x$ and $\beta(\theta)=y$; this defines maps $\alpha, \beta: \Omega \rightarrow M$. Let $1_{x}: E_{x} \rightarrow E_{x}$ denote the identity isometry; then $1: x \mapsto 1_{x}$ maps $M$ to $\Omega$. Finally, let the partial multiplication be composition of maps, and the inverse be the usual inverse of maps. It is immediate that, with these definitions, $\Omega$ is a groupoid.

To give $\Omega$ a smooth structure we modify the technique used in [7, Example 1.1.12]. Let $\left\{U_{\lambda}\right\}$, for $\lambda$ in some indexing set $\Lambda$, be a covering of $M$ by sets of the kind defined in Proposition 5. Let $\tau_{\lambda}: U_{\lambda} \times\left.\mathcal{E} \rightarrow E\right|_{U_{\lambda}}$ be the corresponding isometric local trivialisation of $\left.E\right|_{U_{\lambda}}$, and for $x \in U_{\lambda}$ set $\tau_{\lambda, x}=\tau_{\lambda}(x, \cdot): \mathcal{E} \rightarrow E_{x}$. Let

$$
\Omega_{U_{\mu}, U_{\lambda}}=\bigcup_{x \in U_{\lambda}, y \in U_{\mu}} \Omega_{y, x}
$$


and define

$$
\psi_{\mu, \lambda}: \Omega_{U_{\mu}, U_{\lambda}} \rightarrow U_{\mu} \times \mathfrak{G}(\mathcal{E}) \times U_{\lambda}
$$

where $\mathfrak{G}(\mathcal{E})$ is the isometry group of $\mathcal{E}$ (a Lie group), as follows. If $\theta \in \Omega_{U_{\mu}, U_{\lambda}}$ then $\theta \in \Omega_{y, x}$ for some $x \in U_{\lambda}$ and $y \in U_{\mu}$. Thus $\left(\tau_{\mu, y}\right)^{-1} \circ \theta \circ \tau_{\lambda, x}$ is a map $\mathcal{E} \rightarrow \mathcal{E}$, which is evidently an isometry, say $G \in \mathfrak{G}(\mathcal{E})$. We may therefore put

$$
\psi_{\mu, \lambda}(\theta)=(y, G, x),
$$

and it is straightforward to see that this defines a bijection.

To show that $\Omega$ has a smooth structure, we need to show that if $\psi_{\mu_{2}, \lambda_{2}} \circ$ $\left(\psi_{\mu_{1}, \lambda_{1}}\right)^{-1}$ has a non-empty domain then it is smooth. The non-empty domain condition is that

$$
\left.\left(\psi_{\mu_{1}, \lambda_{1}}\right)^{-1}\left(U_{\mu_{1}} \times \mathfrak{G}(\mathcal{E}) \times U_{\lambda_{1}}\right) \cap \Omega\right|_{U_{\mu_{2}}, U_{\lambda_{2}}} \neq \varnothing
$$

which translates as

$$
\Omega_{U_{\mu_{1}}, U_{\lambda_{1}}} \cap \Omega_{U_{\mu_{2}}, U_{\lambda_{2}}} \neq \varnothing,
$$

in other words that $U_{\lambda_{1}} \cap U_{\lambda_{2}} \neq \varnothing$ and $U_{\mu_{1}} \cap U_{\mu_{2}} \neq \varnothing$. If this condition holds then the domain of $\psi_{\mu_{2}, \lambda_{2}} \circ\left(\psi_{\mu_{1}, \lambda_{1}}\right)^{-1}$ is

$$
\left(U_{\mu_{1}} \cap U_{\mu_{2}}\right) \times \mathfrak{G}(\mathcal{E}) \times\left(U_{\lambda_{1}} \cap U_{\lambda_{2}}\right) .
$$

If $(y, G, x)$ is in the domain then $\left(\psi_{\mu_{1}, \lambda_{1}}\right)^{-1}(y, G, x)$ is the isometry $\tau_{\mu_{1}, y}$ ○ $G \circ\left(\tau_{\lambda_{1}, x}\right)^{-1}: E_{x} \rightarrow E_{y}$. Thus

$$
\begin{gathered}
\psi_{\mu_{2}, \lambda_{2}} \circ\left(\psi_{\mu_{1}, \lambda_{1}}\right)^{-1}(y, G, x)=\psi_{\mu_{2}, \lambda_{2}}\left(\tau_{\mu_{1}, y} \circ G \circ\left(\tau_{\lambda_{1}, x}\right)^{-1}\right) \\
=\left(y,\left(\left(\tau_{\mu_{2}, y}\right)^{-1} \circ \tau_{\mu_{1}, y}\right) \circ G \circ\left(\left(\tau_{\lambda_{1}, x}\right)^{-1} \circ \tau_{\lambda_{2}, x}\right), x\right) .
\end{gathered}
$$

Note that $\left(\tau_{\lambda_{1}, x}\right)^{-1} \circ \tau_{\lambda_{2}, x} \in \mathfrak{G}(\mathcal{E})$, and also $\left(\tau_{\mu_{2}, y}\right)^{-1} \circ \tau_{\mu_{1}, y} \in \mathfrak{G}(\mathcal{E})$, so the central term above is an element of $\mathfrak{G}(\mathcal{E})$, as required. Moreover, $x \mapsto\left(\tau_{\lambda_{1}, x}\right)^{-1} \circ \tau_{\lambda_{2}, x}$ is the transition function $U_{\lambda_{1}} \cap U_{\lambda_{2}} \rightarrow \mathfrak{G}(\mathcal{E})$ for the bundle structure on $\pi: E \rightarrow M$ defined in Proposition 5, and likewise $y \mapsto\left(\tau_{\mu_{1}, y}\right)^{-1} \circ \tau_{\mu_{2}, y}$ is the transition function $U_{\mu_{1}} \cap U_{\mu_{2}} \rightarrow \mathfrak{G}(\mathcal{E})$. So $\psi_{\mu_{2}, \lambda_{2}} \circ\left(\psi_{\mu_{1}, \lambda_{1}}\right)^{-1}$ is constructed from smooth maps by smooth operations, and therefore is smooth.

Now $U_{\lambda}, U_{\mu} \subset M$ are coordinate patches, by assumption. Taking a coordinate patch $V \in \mathfrak{G}(\mathcal{E})$ we obtain a coordinate patch $\left(\psi_{\mu, \lambda}\right)^{-1}\left(U_{\mu} \times\right.$ $\left.V \times U_{\lambda}\right)$ on $\Omega$. The transition functions for such patches are smooth as a 
consequence of the immediately preceding result about $\psi_{\mu_{2}, \lambda_{2}} \circ\left(\psi_{\mu_{1}, \lambda_{1}}\right)^{-1}$. Thus $\Omega$ is a differentiable manifold of dimension $2 m+d, d=\operatorname{dim} \mathfrak{G}(\mathcal{E})$.

It is now straightforward to show that the structure maps of the groupoid are smooth and that the source and target projections are surjective submersions, both separately and as the pair $(\alpha, \beta)$, so we conclude that $\Omega$ is a locally trivial Lie groupoid of dimension $2 m+d$.

We turn now to the Lie algebroid of $\Omega$, which we denote by $A \Omega$. We can identify points of $A \Omega$ over $x \in M$ with tangent vectors at $t=0$ to curves $\kappa$ in the $\alpha$ fibre of $\Omega$ over $x$ such that $\kappa(0)=1_{x}$. For such a curve $\alpha(\kappa(t))=x$, while $\beta(\kappa(t))=\bar{\kappa}(t)$ say is a smooth curve in $M$; so $\kappa(t)$ is an isometry of $E_{x}$ with $E_{\bar{\kappa}(t)}$ and $\kappa(0)$ is the identity map of $E_{x}$. Since $\kappa(t)$ is an isometry, for every $u \in E_{x}$

$$
\kappa(t)^{*} g_{\kappa(t) u}=g_{u}
$$

or in terms of fibre coordinate fields

$$
g_{\kappa(t) u}\left(\kappa(t)_{*} \frac{\partial}{\partial u^{a}}, \kappa(t)_{*} \frac{\partial}{\partial u^{b}}\right)=g_{a b}(u) .
$$

Let us set $\kappa(t) u=\left(\bar{\kappa}^{i}(t), \kappa^{a}(t, u)\right)$; then

$$
\kappa(t)_{*}\left(\left.\frac{\partial}{\partial u^{a}}\right|_{u}\right)=\left.\frac{\partial \kappa^{b}}{\partial u^{a}}(t, u) \frac{\partial}{\partial u^{b}}\right|_{\kappa(t) u},
$$

whence

$$
g_{\kappa(t) u}\left(\kappa(t)_{*} \frac{\partial}{\partial u^{a}}, \kappa(t)_{*} \frac{\partial}{\partial u^{b}}\right)=\frac{\partial \kappa^{c}}{\partial u^{a}}(t, u) \frac{\partial \kappa^{d}}{\partial u^{b}}(t, u) g_{c d}(\kappa(t) u) .
$$

Note that since $\kappa(0)$ is the identity,

$$
\frac{\partial \kappa^{c}}{\partial u^{a}}(0, u)=\delta_{a}^{c} .
$$

The tangent vector $Z_{u}$ to the curve $t \mapsto \kappa(t) u$ at $t=0$ is

$$
\frac{d \bar{\kappa}^{i}}{d t}(0) \frac{\partial}{\partial x^{i}}+\frac{\partial \kappa^{a}}{\partial t}(0, u) \frac{\partial}{\partial u^{a}}=\xi^{i} \frac{\partial}{\partial x^{i}}+\eta^{a}(u) \frac{\partial}{\partial u^{a}}
$$

say. Then $Z$ is a vector field along $E_{x} \rightarrow E$, which projects onto a vector at $x \in M$, namely the initial tangent vector to $\bar{\kappa}$. By differentiating the isometry condition

$$
\frac{\partial \kappa^{c}}{\partial u^{a}}(t, u) \frac{\partial \kappa^{d}}{\partial u^{b}}(t, u) g_{c d}(\kappa(t) u)=g_{a b}(u)
$$


with respect to $t$ and setting $t=0$ we obtain

$$
\xi^{i} \frac{\partial g_{a b}}{\partial x^{i}}+\eta^{c} \frac{\partial g_{a b}}{\partial u^{c}}+g_{c b} \frac{\partial \eta^{c}}{\partial u^{a}}+g_{a c} \frac{\partial \eta^{c}}{\partial u^{b}}=0 .
$$

This is just the condition for $Z$ to belong to $\mathfrak{J}^{x}$.

Conversely, suppose that $Z \in \mathfrak{J}^{x}$. Let $X$ be any vector field on $M$ such that $X_{x}=\pi_{*} Z$. Let $\tilde{X}$ be a vector field on $E$ such that $\tilde{X} \in \mathfrak{I}$ and $\pi_{*} \tilde{X}=X$. Then $\tilde{X}_{x}-Z$ is a vertical vector field on $E_{x}$ belonging to $\mathfrak{J}^{x}$, and is therefore an infinitesimal isometry of $g_{x}$. Let $\tilde{\varphi}_{t}$ be the flow of $\tilde{X}$. By Proposition 3 there is an open interval containing 0 such that $\tilde{\varphi}_{t}: E_{x} \rightarrow E_{\varphi_{t}(x)}$ is an isometry for all $t \in I$, where $\varphi_{t}=\pi \circ \tilde{\varphi}_{t}$. Set $V(t)=\tilde{\varphi}_{t *}\left(\tilde{X}_{x}-Z\right)$ : since $\tilde{\varphi}_{t}$ is an isometry, $V(t)$ is a vertical vector field which is an infinitesimal isometry of $g_{\varphi_{t}(x)}$. Consider $\tilde{X}+V$ : this is a vector field over the curve $\varphi_{t}(x)$ which projects onto $X$ and belongs to $\mathfrak{J}^{\varphi_{t}(x)}$ for all $t \in I$. It generates a curve of isometries $E_{x} \rightarrow E_{\varphi_{t}(x)}$, defined on some open interval containing 0 (possibly smaller than $I$ ), whose tangent at $t=0$ is just $Z$, as required.

When the conditions of Theorem 3 apply we shall denote the Lie algebroid of the fibre-isometry groupoid by $\mathfrak{I}$. Then $\mathfrak{I}$ is a vector bundle over $M$, whose fibre $\mathfrak{I}_{x}$ over $X \in M$ consists of the vector fields along $E_{x} \rightarrow E$ which satisfy the isometry equation at $x$. We denote by $\operatorname{Sec}(\mathfrak{I})$ the $C^{\infty}(M)$-module of sections of $\mathfrak{I}$; elements of $\operatorname{Sec}(\mathfrak{I})$ are vector fields on $E$ which satisfy the isometry equation. The anchor is $\left.\pi_{*}\right|_{\mathfrak{I}}$, and the bracket is just the bracket of vector fields on $E$, restricted of course to elements of $\operatorname{Sec}(\mathfrak{I})$. We denote the kernel by $\mathfrak{K} \rightarrow M$; it is a Lie algebra bundle, and for each $x \in M$ the fibre $\mathfrak{K}_{x}$ is the Lie algebra of infinitesimal isometries of the Riemannian manifold $\left(E_{x}, g_{x}\right)$.

We have the following short exact sequence of vector bundles over $M$ :

$$
0 \rightarrow \mathfrak{K} \rightarrow \mathfrak{I} \rightarrow T M \rightarrow 0 .
$$

We now specialise further to the case where this sequence splits; that is to say, we suppose that there is a linear bundle map $\gamma: T M \rightarrow \mathfrak{I}$ over the identity of $M$ such that $\pi_{*} \circ \gamma=\mathrm{id}_{T M}$. Such a splitting is an infinitesimal connection on $\mathfrak{I}$, in the terminology of Section 2. There is a corresponding connection $\Gamma$ on the fibre-isometry groupoid. We now set out to identify its holonomy Lie algebra bundle.

Proposition 6. Suppose we have an infinitesimal connection $\gamma$ on $\mathfrak{I}$. For any $X \in \mathfrak{X}(M)$ and $V \in \operatorname{Sec}(\mathfrak{K})$ set

$$
\nabla_{X}^{\gamma} V=[\gamma(X), V]
$$


Then $\nabla^{\gamma}$ is a covariant differentiation operator on $\operatorname{Sec}(\mathfrak{K})$. Furthermore, $\nabla_{X}^{\gamma}$ is a derivation of the bracket:

$$
\nabla_{X}^{\gamma}[V, W]=\left[\nabla_{X}^{\gamma} V, W\right]+\left[V, \nabla_{X}^{\gamma} W\right] .
$$

Proof. Since $\gamma(X) \in \operatorname{Sec}(\mathfrak{I})$ and $\operatorname{Sec}(\mathfrak{I})$ is closed under bracket, $\nabla_{X}^{\gamma} V \in$ $\operatorname{Sec}(\mathfrak{I})$. But $\nabla_{X}^{\gamma} V$ is clearly vertical, so $\nabla_{X}^{\gamma} V \in \operatorname{Sec}(\mathfrak{K})$. Evidently $\nabla_{X}^{\gamma} V$ is $\mathbb{R}$-linear in both arguments, and for $f \in C^{\infty}(M)$,

$$
\nabla_{f X}^{\gamma} V=f \nabla_{X}^{\gamma} V, \quad \nabla_{X}^{\gamma}(f V)=f \nabla_{X}^{\gamma} V+X(f) V .
$$

The fact that $\nabla_{X}^{\gamma}$ is a derivation of the bracket follows from the Jacobi identity.

The conclusion that $\nabla_{X}^{\gamma} V \in \operatorname{Sec}(\mathfrak{K})$ for all $X \in \mathfrak{X}(M)$ and $V \in \operatorname{Sec}(\mathfrak{K})$ is abbreviated to $\nabla^{\gamma}(\operatorname{Sec}(\mathfrak{K})) \subset \operatorname{Sec}(\mathfrak{K})$.

A connection for which the derivation property is satisfied is called a Lie connection. It is a consequence of the fact that the connection is Lie that the parallel translation operator $\tau_{c}^{\gamma}$ corresponding to $\nabla^{\gamma}$ along a curve $c$ in $M$ from $x$ to $y$ is an isomorphism of Lie algebras $\mathfrak{K}_{x} \rightarrow \mathfrak{K}_{y}$ (see Proposition 13 in Appendix 2).

For any $X, Y \in \mathfrak{X}(M)$ we set

$$
R^{\gamma}(X, Y)=\gamma([X, Y])-[\gamma(X), \gamma(Y)]
$$

Then $R^{\gamma}(X, Y)$ is a vertical vector field on $E$ which depends $C^{\infty}(M)$-linearly on $X$ and $Y$, so for any $x \in M$ and any $v, w \in T_{x} M, R_{x}^{\gamma}(v, w)$ is a welldefined vertical vector field on the fibre $E_{x}$. We call $R^{\gamma}$ the curvature of $\gamma$ and we call $R_{x}^{\gamma}(v, w)$ a curvature vector field at $x$.

Proposition 7. For all $X, Y \in \mathfrak{X}(M), R^{\gamma}(X, Y) \in \operatorname{Sec}(\mathfrak{K})$.

Proof. Since $\operatorname{Sec}(\mathfrak{I})$ is closed under bracket, $[\gamma(X), \gamma(Y)] \in \operatorname{Sec}(\mathfrak{I})$. Thus both $\gamma([X, Y])$ and $[\gamma(X), \gamma(Y)]$ belong to $\operatorname{Sec}(\mathfrak{I})$; their difference, which is vertical, therefore belongs to $\operatorname{Sec}(\mathfrak{K})$.

Corollary 1. For every $x \in M$ and $v, w \in T_{x} M, R_{x}^{\gamma}(v, w) \in \mathfrak{K}_{x}$.

We can also compute the curvature of $\nabla^{\gamma}$; we obtain

$$
\begin{aligned}
\nabla_{X}^{\gamma} \nabla_{Y}^{\gamma} V-\nabla_{Y}^{\gamma} \nabla_{X}^{\gamma} V-\nabla_{[X, Y]}^{\gamma} V & =[V, \gamma([X, Y])-[\gamma(X), \gamma(Y)]] \\
& =\left[V, R^{\gamma}(X, Y)\right] .
\end{aligned}
$$


Due to the existence of the splitting we have $\mathfrak{I} \equiv T M \oplus \mathfrak{K}$. Thus sections of $\mathfrak{I} \rightarrow M$ are vector fields on $E$ of the form $\gamma(X)+V$ with $V \in \operatorname{Sec}(\mathfrak{K})$. In terms of the direct sum decomposition the algebroid bracket is given by

$$
[X \oplus V, Y \oplus W]=[X, Y] \oplus\left(-R^{\gamma}(X, Y)+\nabla_{X}^{\gamma} W-\nabla_{Y}^{\gamma} V+[V, W]\right) .
$$

Every term in the second component on the right belongs to $\operatorname{Sec}(\mathfrak{K})$.

By Theorem 2, the holonomy Lie algebra bundle is the least Lie algebra sub-bundle $\mathfrak{H}$ of $\mathfrak{K}$ which contains all curvature vector fields (that is, such that for all $x \in M$ and all $\left.v, w \in T_{x} M, R_{x}^{\gamma}(v, w) \in \mathfrak{H}_{x}\right)$, and satisfies $\nabla^{\gamma}(\operatorname{Sec}(\mathfrak{H})) \subset \operatorname{Sec}(\mathfrak{H})$. (That there is a least Lie algebra sub-bundle with these properties is a consequence of the following result, whose proof is to be found in Appendix 2, Corollary 4: let $E \rightarrow M$ be a Lie algebra bundle equipped with a Lie connection, and let $E^{1}$ and $E^{2}$ be Lie algebra subbundles of $E$ such that $\nabla\left(\operatorname{Sec}\left(E^{i}\right)\right) \subset \operatorname{Sec}\left(E^{i}\right), i=1,2$; then $E^{1} \cap E^{2}$ is a Lie algebra bundle.) The holonomy algebra at $x$ is $\mathfrak{H}_{x}$.

We now prove a version of the Ambrose-Singer Theorem in the present context. For this purpose we define, for every $x \in M$, a vector subspace $\mathfrak{R}_{x}$ of the vector space $\mathfrak{K}_{x}$, as follows. Since for any $y \in M$ and any curve $c$ in $M$ joining $y$ to $x$ parallel translation $\tau_{c}^{\gamma}$ along $c$ maps $\mathfrak{K}_{y}$ to $\mathfrak{K}_{x}, \tau_{c}^{\gamma} R_{y}^{\gamma}(v, w) \in \mathfrak{K}_{x}$ for any $v, w \in T_{y} M$. We define $\mathfrak{R}_{x}$ to be the least subspace of $\mathfrak{K}_{x}$ containing all parallel translates of curvature vector fields to $x$, that is, all $\tau_{c}^{\gamma} R_{y}^{\gamma}(v, w)$.

Theorem 4. $\mathfrak{H}_{x}=\mathfrak{R}_{x}$.

In the proof we use the following result. Let $\pi: E \rightarrow M$ be a vector bundle equipped with a linear connection $\nabla$. Let $E^{\prime}$ be a subset of $E$ such that: $\pi$ maps $E^{\prime}$ onto $M$; for each $x \in M, E_{x}^{\prime}=\left.\pi\right|_{E^{\prime}} ^{-1}(x)$ is a linear subspace of $E_{x}$; and for any pair of points $x, y \in M$ and any curve $c$ in $M$ joining them, $\tau_{c}\left(E_{x}^{\prime}\right) \subseteq E_{y}^{\prime}$. Then $E^{\prime}$ is a vector sub-bundle of $E$ and $\nabla\left(\operatorname{Sec}\left(E^{\prime}\right)\right) \subset$ $\operatorname{Sec}\left(E^{\prime}\right)$. The proof is to be found in Appendix 2, Proposition 12.

Proof. Set $\mathfrak{R}=\bigcup_{x} \mathfrak{R}_{x}$. It follows from Proposition 12 (with $E=\mathfrak{K}$ and $\left.E^{\prime}=\mathfrak{R}\right)$ that $\mathfrak{R}$ is a vector sub-bundle of $\mathfrak{K}$, and $\nabla^{\gamma}(\operatorname{Sec}(\mathfrak{R})) \subset \operatorname{Sec}(\mathfrak{R})$. Thus for any $V \in \operatorname{Sec}(\mathfrak{R})$ and any $X, Y \in \mathfrak{X}(M)$,

$$
\nabla_{X}^{\gamma} \nabla_{Y}^{\gamma} V-\nabla_{Y}^{\gamma} \nabla_{X}^{\gamma} V-\nabla_{[X, Y]}^{\gamma} V=\left[V, R^{\gamma}(X, Y)\right] \in \operatorname{Sec}(\Re) .
$$

Thus $\left[\tau_{c}^{\gamma} R_{y}^{\gamma}\left(v_{y}, w_{y}\right), R_{x}^{\gamma}\left(v_{x}, w_{x}\right)\right] \in \mathfrak{R}_{x}$ for any $v_{x}, w_{x} \in T_{x} M$ and $v_{y}, w_{y} \in$ $T_{y} M$. But since $\nabla^{\gamma}$ is a Lie connection, parallel translation preserves brackets (Proposition 13), so for any $y, z \in M$, any curve $c$ in $M$ joining $y$ to $x$ and any curve $d$ in $M$ joining $z$ to $x,\left[\tau_{c}^{\gamma} R_{y}^{\gamma}\left(v_{y}, w_{y}\right), \tau_{d}^{\gamma} R_{z}^{\gamma}\left(v_{z}, w_{z}\right)\right] \in \mathfrak{R}_{x}$. 
Thus $\mathfrak{R}_{x}$ is a Lie subalgebra of $\mathfrak{K}_{x}$. Moreover, the Lie algebras $\mathfrak{R}_{x}$ at different points are isomorphic, so $\mathfrak{R}$ is a Lie algebra sub-bundle of $\mathfrak{K}$, and as we have pointed out already, $\nabla^{\gamma}(\operatorname{Sec}(\mathfrak{R})) \subset \operatorname{Sec}(\mathfrak{R})$. Evidently $R_{x}^{\gamma}(v, w) \in \mathfrak{R}_{x}$ for every $v, w \in T_{x} M$. It follows that $\mathfrak{H} \subseteq \mathfrak{R}$. On the other hand, any Lie algebra sub-bundle $\mathfrak{K}^{\prime}$ of $\mathfrak{K}$ which contains all curvature vector fields and satisfies $\nabla^{\gamma}\left(\operatorname{Sec}\left(\mathfrak{K}^{\prime}\right)\right) \subset \operatorname{Sec}\left(\mathfrak{K}^{\prime}\right)$ must contain all the parallel translates of curvature vector fields, and so must contain $\mathfrak{R}$. Thus $\mathfrak{R} \subseteq \mathfrak{H}$, and so $\mathfrak{R}=\mathfrak{H}$. Thus $\mathfrak{R}_{x}=\mathfrak{H}_{x}$.

\section{Holonomy of Landsberg spaces}

We now discuss the application of the results of the previous section to Landsberg spaces in Finsler geometry. (Some earlier and much more rudimentary steps in this direction are to be found in [4].)

It is well known $[1,2,10]$ that a Finsler space over a manifold $M$ supports a canonical nonlinear connection or horizontal distribution, that is, a distribution on the slit tangent bundle $\pi: T^{\circ} M \rightarrow M$ which is everywhere transverse to the fibres. (The term 'connection' has two distinct uses in this section - a second one will be introduced below - not to mention the uses in the context of the groupoid theory. We therefore prefer the term horizontal distribution since it reduces the possibilities of confusion.) We shall not need the explicit definition of the horizontal distribution, only the fact that it exists, that it is positively-homogeneous, and that it enjoys one or two other properties to be introduced in due course.

Suppose given a Finsler space, together with its canonical horizontal distribution. A smooth curve in $T^{\circ} M$ is horizontal if its tangent vector is everywhere horizontal; the definition extends to piecewise-smooth curves in the obvious way. Let $c$ be a piecewise-smooth curve in $M, x$ a point in the image of $c$ and $u \in T_{x}^{\circ} M$. A piecewise-smooth curve in $T^{\circ} M$ which is horizontal, projects onto $c$ and passes through $(x, u)$ is called a horizontal lift of $c$ through $u$. If $c$ is defined on the finite closed interval $[a, b]$ and $u \in T_{x}^{\circ} M, x=c(a)$, then there is a unique horizontal lift of $c$ through $u$ which is also defined on $[a, b]$. If one makes a reparametrization of $c$, carrying out the same reparametrization on its horizontal lift produces a horizontal lift of the reparametrization of $c$. As a consequence of this reparametrization property we may standardize the domains of definition of curves to $[0,1]$. We may therefore make the definition of the horizontal lift more specific, as follows. Let $c:[0,1] \rightarrow M$ be any piecewise-smooth curve, with $c(0)=x$. For any $u \in T_{x}^{\circ} M$ the horizontal lift of $c$ to $u$ is the unique piecewise-smooth 
horizontal curve $c_{u}^{\mathrm{H}}:[0,1] \rightarrow T^{\circ} M$ such that $\pi \circ c_{u}^{\mathrm{H}}=c$ and $c_{u}^{\mathrm{H}}(0)=u$.

For any $x, y \in M$ and any piecewise-smooth curve $c$ with $c(0)=x$, $c(1)=y$, we may define a map $\rho_{c}: T_{x}^{\circ} M \rightarrow T_{y}^{\circ} M$ by setting $\rho_{c}(u)=c_{u}^{\mathrm{H}}(1)$, which is in fact a diffeomorphism. (The map $\rho_{c}$ is sometimes called nonlinear parallel transport [1], but again we prefer to avoid this term for the sake of clarity.) Slightly more generally, for $t \in[0,1]$ we define $\rho_{c}(t): T_{x}^{\circ} M \rightarrow T_{c(t)}^{\circ} M$ by $\rho_{c}(t)(u)=c_{u}^{\mathrm{H}}(t)$.

The canonical horizontal distribution of a Finsler space has the property that the Finsler function $F$ is constant along horizontal curves. Because of this, and because the horizontal distribution is positively-homogeneous, we may restrict our attention to the indicatrix bundle $\mathcal{I}=\left\{(x, u) \in T^{\circ} M\right.$ : $F(x, u)=1\}$; this is a fibre bundle over $M$ with compact fibres (each indicatrix is diffeomorphic to a sphere). In particular, each $\rho_{c}$ restricts to a diffeomorphism of indicatrices, $\rho_{c}: \mathcal{I}_{x} \rightarrow \mathcal{I}_{y}$.

For any vector field $X$ on $M$ we denote by $X^{\mathrm{H}}$ its horizontal lift to $T^{\circ} M$ with respect to the canonical horizontal distribution; it is the unique horizontal vector field such that $\pi_{*} X^{\mathrm{H}}=X$. Another version of the constancy property is that for every $X \in \mathfrak{X}(M), X^{\mathrm{H}}(F)=0$; in particular, $X^{\mathrm{H}}$ is tangent to $\mathcal{I}$.

In any Finsler space the fundamental tensor $g$ defines a fibre metric on $T^{\circ} M$, and by restriction one on $\mathcal{I}$. A Landsberg space, according to one possible definition (see for example [1]), is a Finsler space for which, for every pair of points $x, y \in M$ and every curve $c$ joining $x$ and $y, \rho_{c}$ is an isometry of the Riemannian spaces $\left(\mathcal{I}_{x}, g_{x}\right)$ and $\left(\mathcal{I}_{y}, g_{y}\right)$. In the light of the previous section we may put things another way: a Finsler space is a Landsberg space if the holonomy groupoid of its canonical horizontal lift, acting on its indicatrix bundle, is a subgroupoid of the fibre-isometry groupoid. Then the connection $\Gamma$ associated with the canonical horizontal lift is just $c^{\Gamma}(t)=\rho_{c}(t)$.

There is an equivalent differential version of this definition. A Landsberg space is one for which $\mathcal{L}_{X^{\mathrm{H}}} g=0$, or in other words $X^{\mathrm{H}} \in \mathfrak{J}$, for all $X \in$ $\mathfrak{X}(M)$. It is certainly the case, therefore, that for a Landsberg space $\mathfrak{J}$ is transitive, and we conclude from Theorem 3 that the fibre-isometry groupoid is a Lie groupoid. We denote by $\mathfrak{I}$ its Lie algebroid, as before. It is moreover the case that the horizontal lift defines an infinitesimal connection on $\mathfrak{I}$ : for $v \in T_{x} M$ we define $\gamma(v)=v^{\mathrm{H}} \in \mathfrak{I}_{x}$ (where $v^{\mathrm{H}}$ is considered as a vector field along $\mathcal{I}_{x} \rightarrow \mathcal{I}$ satisfying the isometry equation at $x$ ).

The theory of the previous section applies to Landsberg spaces, therefore. There is one point of interest which deserves closer inspection, and that con- 
cerns the identification of the covariant derivative operator $\nabla^{\gamma}$. To discuss this point it will be worthwhile to revert temporarily to the consideration of the canonical horizontal distribution as a distribution on $T^{\circ} M$.

We may associate with every vector field $X$ on $M$ an operator $\nabla_{X}$ on vertical vector fields $V$ on $T^{\circ} M$ by

$$
\nabla_{X} V=\left[X^{\mathrm{H}}, V\right]
$$

Then $\nabla_{X} V$ is vertical, is $\mathbb{R}$-linear in both arguments, and for $f \in C^{\infty}(M)$

$$
\nabla_{f X} V=f \nabla_{X} V, \quad \nabla_{X}(f V)=f \nabla_{X} V+X(f) V ;
$$

so $\nabla$ has the properties of a (linear) covariant differentiation operator. Furthermore, $\nabla_{X}$ is a derivation of the bracket:

$$
\nabla_{X}[V, W]=\left[\nabla_{X} V, W\right]+\left[V, \nabla_{X} W\right]
$$

by the Jacobi identity. Of course, to call this a covariant derivative would be stretching a point, since to do so we should have to regard the space of vertical vector fields on $T_{x}^{\circ} M$ as the fibre of a vector bundle over $M$, and a vertical vector field on $T^{\circ} M$ as a section of this bundle.

If the canonical horizontal distribution is spanned locally by vector fields

$$
H_{i}=\frac{\partial}{\partial x^{i}}-\Gamma_{i}^{j} \frac{\partial}{\partial u^{j}}
$$

then

$$
\nabla_{\partial / \partial x^{i}}\left(\frac{\partial}{\partial u^{j}}\right)=\left[H_{i}, \frac{\partial}{\partial u^{j}}\right]=\frac{\partial \Gamma_{i}^{k}}{\partial u^{j}} \frac{\partial}{\partial u^{k}} .
$$

Now the connection coefficients $\Gamma_{i j}^{k}$ of the Berwald connection are given by

$$
\frac{\partial \Gamma_{i}^{k}}{\partial u^{j}}=\Gamma_{i j}^{k} .
$$

That is to say, $\nabla$ is closely related to the Berwald connection. The Berwald connection is usually thought of as a connection on the vector bundle $\pi^{*} T M$, the pullback of $T M$ over $T^{\circ} M$; it is of course a linear connection. But for us it will be more convenient to work with vertical vector fields on $T^{\circ} M$ than with sections of $\pi^{*} T M$, though there is no essential difference (every section of $\pi^{*} T M$ defines a vertical vector field on $T^{\circ} M$ by the vertical lift procedure, and the correspondence is 1-1). In fact for a curve $c$ on $M, \nabla_{\dot{c}}$ is effectively covariant differentiation with respect to the Berwald connection along horizontal lifts of $c$. 
There is a further point of interest, concerned with the differential of the map $\rho_{c}$ for a curve $c$ joining $x$ and $y$ in $M$. What we have to say initially applies to any Finsler space, and it will continue to be convenient initially to consider the action of $\rho_{c}$ on $T^{\circ} M$. Since $T_{x}^{\circ} M$ is a vector space (though deprived of its origin), its tangent space at any point may be canonically identified with itself (with origin restored); so for any $u \in T_{x}^{\circ} M, \rho_{c * u}$ may be regarded as a (linear) map $T_{x} M \rightarrow T_{y} M$. This map may be described as follows. For $v \in T_{x} M, u \in T_{x}^{\circ} M$, let $v(t)$ be the (unique) vector field along $c_{u}^{\mathrm{H}}$ which is parallel with respect to the Berwald connection of the Finsler space and satisfies $v(0)=v$ : then $\rho_{c * u}(v)=v(1)$. This may be seen as follows. Consider the pullback $c^{*} T^{\circ} M$. The canonical horizontal distribution on $T^{\circ} M$ induces one on $c^{*} T^{\circ} M$, which is 1-dimensional and is spanned by the vector field

$$
\left(\frac{\partial}{\partial t}\right)^{\mathrm{H}}=\frac{\partial}{\partial t}-\dot{c}^{j} \Gamma_{j}^{i}\left(c^{k}, u^{k}\right) \frac{\partial}{\partial u^{i}} .
$$

In view of the definition of $\rho_{c}$ it is clear that $\rho_{c * u}(v)$ is the Lie translate by $(\partial / \partial t)^{\mathrm{H}}$ of $v$, considered as a vertical vector at $u$, to $\rho_{c}(u)$. For any vertical vector field

$$
V=V^{i}\left(t, u^{k}\right) \frac{\partial}{\partial u^{i}}
$$

on $c^{*} T^{\circ} M$,

$$
\begin{aligned}
{\left[\left(\frac{\partial}{\partial t}\right)^{\mathrm{H}}, V^{i} \frac{\partial}{\partial u^{i}}\right] } & =\left(\left(\frac{\partial}{\partial t}\right)^{\mathrm{H}}\left(V^{i}\right)+\Gamma_{j k}^{i} \dot{c}^{j} V^{k}\right) \frac{\partial}{\partial u^{i}} \\
& =\left(\dot{c}_{u}^{\mathrm{H}}\left(V^{i}\right)+\Gamma_{j k}^{i} \dot{c}^{j} V^{k}\right) \frac{\partial}{\partial u^{i}} .
\end{aligned}
$$

Thus $V$ is Lie transported by $(\partial / \partial t)^{\mathrm{H}}$ if and only if

$$
\dot{c}_{u}^{\mathrm{H}}\left(V^{i}\right)+\Gamma_{j k}^{i} \dot{c}^{j} V^{k}=0,
$$

that is, if and only if it is parallelly translated along $c_{u}^{\mathrm{H}}$ with respect to the Berwald connection. We can conveniently summarise this in terms of $\nabla$, as follows. For any curve $c$ on $M$ with $c(0)=x, c(1)=y$, the map $\rho_{c *}$, considered as a map from vertical vector fields on $T_{x}^{\circ} M$ to vertical vector fields on $T_{y}^{\circ} M$, is given by parallel translation with respect to $\nabla$, in the sense that if $V(t)$ is a field of vertical vector fields along $c(t)$ with $\nabla_{\dot{c}} V=0$, then $\rho_{c *} V(0)=V(1)$.

We can formally compute the curvature of $\nabla$; we obtain

$$
\nabla_{X} \nabla_{Y} V-\nabla_{Y} \nabla_{X} V-\nabla_{[X, Y]} V=[V, R(X, Y)]
$$


where $R$ is the curvature of the canonical horizontal distribution; note that $R(X, Y)$ is a vertical vector field.

One version of the Landsberg property is that $\mathcal{L}_{X^{\mathrm{H}}} g=0$ for all $X \in$ $\mathfrak{X}(M)$. But

$$
\begin{aligned}
\mathcal{L}_{X^{\mathrm{H}}} g(V, W) & =X^{\mathrm{H}}(g(V, W))-g\left(\left[X^{\mathrm{H}}, V\right], W\right)-g\left(V,\left[X^{\mathrm{H}}, W\right]\right) \\
& =X^{\mathrm{H}}(g(V, W))-g\left(\nabla_{X} V, W\right)-g\left(V, \nabla_{X} W\right)
\end{aligned}
$$

so if we extend the action of $\nabla$ to fibre metrics in the obvious way we may write the condition as $\nabla g=0$.

We return to consideration of $\mathcal{I}$, the Lie algebroid $\mathfrak{I}$ and its kernel $\mathfrak{K}$, in a Landsberg space. We now consider $X^{\mathrm{H}}$, for any $X \in \mathfrak{X}(M)$, as a vector field on $\mathcal{I}$; then in a Landsberg space we have $X^{\mathrm{H}} \in \operatorname{Sec}(\mathfrak{I})$. The infinitesimal connection is just $\gamma(X)=X^{\mathrm{H}}$, and $\nabla^{\gamma}$ is the restriction of the operator $\nabla$ to $\operatorname{Sec}(\mathfrak{K})$. Since $\mathfrak{K}$ is a vector bundle the restriction of $\nabla$ is a genuine covariant derivative. From the theory of the previous section we deduce the following proposition (which may also be proved directly from the definitions of $\mathfrak{K}$ and $\nabla)$.

Proposition 8. In a Landsberg space

1. $\nabla(\operatorname{Sec}(\mathfrak{K})) \subset \operatorname{Sec}(\mathfrak{K})$;

2. for all $X, Y \in \mathfrak{X}(M), R(X, Y) \in \operatorname{Sec}(\mathfrak{K})$.

We can now apply the results of the previous section to the case of a Landsberg space. We have a locally trivial Lie groupoid, the fibre-isometry groupoid of $\mathcal{I}$, whose Lie algebroid $\mathfrak{I}$ is equipped with an infinitesimal connection $\gamma: T M \rightarrow \mathfrak{I}$, where for $v \in T_{x} M, \gamma(v)=v^{\mathrm{H}}$. The corresponding connection $\Gamma$ is the map $c \mapsto \rho_{c}$ (where $\rho_{c}$ is regarded as a curve in the fibreisometry groupoid). The covariant derivative operator $\nabla^{\gamma}$ corresponding to $\gamma$, which acts on sections of the kernel, is just $\nabla$ operating on $\operatorname{Sec}(\mathfrak{K})$. The holonomy groupoid is a Lie groupoid, and the holonomy Lie algebra bundle is the least Lie algebra sub-bundle $\mathfrak{H}$ of $\mathfrak{K}$ which contains all curvature vector fields $R_{x}(v, w)$ for $x \in M$ and $v, w \in T_{x} M$ and satisfies $\nabla(\operatorname{Sec}(\mathfrak{H})) \subset \operatorname{Sec}(\mathfrak{H})$. We may further specialise the Ambrose-Singer Theorem, Theorem 4, from the previous section. For this purpose we recall that the parallel translation operator corresponding to $\nabla$ along a curve $c$ from $y$ to $x$ is the restriction to $\mathfrak{K}_{y}$ of $\rho_{c *}$, the differential of $\rho_{c}$. This is an isomorphism of Lie algebras $\mathfrak{K}_{y} \rightarrow \mathfrak{K}_{x}$, as follows from the general theory, and also directly from the fact that $\rho_{c}$ is an isometry. We now define $\mathfrak{R}_{x}$ to be the least subspace of $\mathfrak{K}_{x}$ containing all elements of the form $\rho_{c *} R_{y}(v, w)$.

Theorem 5. In a Landsberg space $\mathfrak{H}_{x}=\mathfrak{R}_{x}$. 


\section{Covariant derivatives of the curvature}

This section is devoted to clarifying the relationship between the holonomy algebra $\mathfrak{H}_{x}$ of a Landsberg space and the algebra generated by the curvature and its covariant derivatives. It is based on Section 10 of Chapter II of Kobayashi and Nomizu [5].

We define, for each $k=2,3, \ldots$, an $\mathbb{R}$-linear space of vertical vector fields $\mathfrak{C}^{k}$ on $\mathcal{I}$ inductively as follows: $\mathfrak{C}^{2}$ is the set of all finite linear combinations over $\mathbb{R}$ of vertical vector fields of the form $R(X, Y)$ for any pair of vector fields $X, Y$ on $M ; \mathfrak{C}^{k}$ is the set of all finite linear combinations over $\mathbb{R}$ of vertical vector fields which either belong to $\mathfrak{C}^{k-1}$ or are of the form $\nabla_{X} V$ for some vector field $X$ on $M$, where $V \in \mathfrak{C}^{k-1}$.

Theorem 6. With $\mathfrak{C}^{k}$ defined as above

1. each $\mathfrak{C}^{k}$ is a $C^{\infty}(M)$-module;

2. $\left[\mathfrak{C}^{k}, \mathfrak{C}^{l}\right] \subset \mathfrak{C}^{k+l}$;

3. for $m=0,1,2, \ldots, \mathfrak{C}^{m+2} / \mathfrak{C}^{m+1}$ is spanned by equivalence classes of vertical vector fields of the form

$$
\nabla_{X_{1}} \nabla_{X_{2}} \cdots \nabla_{X_{m}}(R(Y, Z))
$$

where $X_{1}, X_{2}, \ldots, X_{m}, Y$ and $Z$ are any vector fields on $M$ (and $\left.\mathfrak{C}^{1}=\{0\}\right)$.

(Note that in the third assertion, every $\nabla$ operates on a vertical vector field. There is no question of writing this formula in terms of covariant differentials of the curvature tensor, as one would for a linear connection, essentially because (for example) $\nabla_{X_{a}} Y$ would make no sense in this context.)

Proof. (1) We have to show that $\mathfrak{C}^{k}$ is closed under scalar multiplication by smooth functions on $M$. For any $f \in C^{\infty}(M), f R(X, Y)=R(f X, Y) \in \mathfrak{C}^{2}$. Suppose that $\mathfrak{C}^{k-1}$ is closed under scalar multiplication by smooth functions on $M$. Then for any $V \in \mathfrak{C}^{k-1}$ we know that $f V \in \mathfrak{C}^{k-1} \subset \mathfrak{C}^{k}$, and $f \nabla_{X} V=\nabla_{f X} V \in \mathfrak{C}^{k}$; so $\mathfrak{C}^{k}$ is closed under scalar multiplication by smooth functions on $M$.

(2) We show first that $\left[\mathfrak{C}^{k}, \mathfrak{C}^{2}\right] \subset \mathfrak{C}^{k+2}$ for all $k \geq 2$. We have

$$
[V, R(X, Y)]=\nabla_{X} \nabla_{Y} V-\nabla_{Y} \nabla_{X} V-\nabla_{[X, Y]} V
$$


and if $V \in \mathfrak{C}^{k}$, each term on the right-hand side belongs to $\mathfrak{C}^{k+2}$. Now suppose that $\left[\mathfrak{C}^{k}, \mathfrak{C}^{l}\right] \subset \mathfrak{C}^{k+l}$ for all $k$ and all $l<L$. Let $V \in \mathfrak{C}^{L}$. It will be sufficient to consider $V=\nabla_{X} W$ with $W \in \mathfrak{C}^{L-1}$. Then

$$
[U, V]=\left[U, \nabla_{X} W\right]=\nabla_{X}[U, W]-\left[\nabla_{X} U, W\right]
$$

For $U \in \mathfrak{C}^{k}$, by the induction assumption $[U, W] \in \mathfrak{C}^{k+L-1}$, so $\nabla_{X}[U, W] \in$ $\mathfrak{C}^{k+L}$; while $\nabla_{X} U \in \mathfrak{C}^{k+1}$, so by the induction assumption again $\left[\nabla_{X} U, W\right] \in$ $\mathfrak{C}^{k+L}$.

(3) Obvious.

Let us set $\bigcup_{k} \mathfrak{C}^{k}=\mathfrak{C}$. Then $\mathfrak{C}$ is a $C^{\infty}(M)$-linear space of vertical vector fields on $\mathcal{I}$ contained in $\operatorname{Sec}(\mathfrak{K})$, which is closed under bracket, contains $R(X, Y)$ and is invariant under $\nabla$; and it is minimal with respect to these properties. It is clear, moreover, that $\mathfrak{C}^{k} \subset \operatorname{Sec}(\mathfrak{H})$ for all $k=2,3, \ldots$ so $\mathfrak{C} \subset \operatorname{Sec}(\mathfrak{H})$. However, we have no guarantee that $\mathfrak{C}$ consists of the sections of a Lie algebra bundle, so in particular we cannot identify it with $\operatorname{Sec}(\mathfrak{H})$.

For $x \in M$ we set $\mathfrak{C}_{x}=\left\{V_{x}: V \in \mathfrak{C}\right\}$. Then $\mathfrak{C}_{x}$ consists of vertical vector fields on $\mathcal{I}_{x}$, and is a Lie algebra with respect to the bracket of vertical vector fields. Clearly, $\mathfrak{C}_{x}$ is a Lie subalgebra of the holonomy algebra $\mathfrak{H}_{x}$ for each $x \in M$. However, we have no reason to suppose that $\mathfrak{C}_{x}$ and $\mathfrak{C}_{y}$ are isomorphic Lie algebras for $x \neq y$, or even that they have the same dimension.

Proposition 9. For each $x \in M$ there is a neighbourhood $U$ of $x$ in $M$ such that for all $y \in U, \operatorname{dim} \mathfrak{C}_{y} \geq \operatorname{dim} \mathfrak{C}_{x}$.

Proof. Every element of $\mathfrak{C}_{x}$ is the restriction to $\mathcal{I}_{x}$ of an element of $\mathfrak{C}$. Let $V_{a}, a=1,2, \ldots, \operatorname{dim} \mathfrak{C}_{x}$, be elements of $\mathfrak{C}^{\mathfrak{s}}$ such that $\left\{\left.V_{a}\right|_{x}\right\}$ is a basis for $\mathfrak{C}_{x}$. Since the $\left.V_{a}\right|_{x}$ are linearly independent, there is a neighbourhood $U$ of $x$ such that the $\left.V_{a}\right|_{y}$ are linearly independent for all $y \in U$. Then $\operatorname{dim} \mathfrak{C}_{y} \geq \operatorname{dim} \mathfrak{C}_{x}$ for all $y \in U$.

Proposition 10. Let $\mu$ be the maximum value of $\operatorname{dim} \mathfrak{C}_{x}$ as $x$ ranges over $M$. Then the set $\left\{x \in M: \operatorname{dim} \mathfrak{C}_{x}=\mu\right\}$ is an open subset of $M$. Let $U$ be a path-connected component of it: then for each $x \in U, \mathfrak{C}_{x}$ is the holonomy algebra at $x$ of the Landsberg space defined over $U$ by the restriction of the Landsberg Finsler function $F$ to $T^{\circ} U$.

Proof. By Proposition 9, every point $x$ with $\operatorname{dim} \mathfrak{C}_{x}=\mu$ has a neighbourhood at all of whose points $y, \operatorname{dim} \mathfrak{C}_{y}=\mu$; so $\left\{x \in M: \operatorname{dim} \mathfrak{C}_{x}=\mu\right\}$ is open. Let $U$ be a path-connected component of this set, and restrict both the Landsberg 
structure and $\mathfrak{C}$ to this open submanifold of $M$. If $V_{a}, a=1,2, \ldots, \mu$, are elements of $\left.\mathfrak{C}\right|_{U}$ such that $\left\{\left.V_{a}\right|_{x}\right\}$ is a basis for $\mathfrak{C}_{x}$ for some $x \in U$, then they also form a basis for $\mathfrak{C}_{y}$ for all $y$ in some neighbourhood of $x$ in $U$; so $\bigcup_{x \in U} \mathfrak{C}_{x}$ is a vector bundle over $U$, and $\left.\mathfrak{C}\right|_{U}$ consists of its sections. Each of its fibres is a Lie algebra, and $\nabla$ is a Lie connection for the corresponding bracket of sections: so it is a Lie algebra bundle. We denote it by $\overline{\mathfrak{K}}$. Now $\overline{\mathfrak{K}}$ evidently contains all curvature vector fields $R_{x}(v, w)$ for $x \in U, v, w \in T_{x} U$, and of course $\nabla(\operatorname{Sec}(\overline{\mathfrak{K}})) \subset \operatorname{Sec}(\overline{\mathfrak{K}})$. Moreover, by construction $\overline{\mathfrak{K}}$ is contained in any Lie algebra sub-bundle of $\left.\mathfrak{K}\right|_{U}$ with these properties. It is therefore the least such Lie algebra bundle, which is to say that it is the holonomy Lie algebra bundle of the restriction of the Landsberg structure to $U$.

Corollary 2. If $\operatorname{dim} \mathfrak{C}_{x}$ is constant on $M$ then $\overline{\mathfrak{K}}=\mathfrak{H}$, and $\mathfrak{C}_{x}=\mathfrak{H}_{x}$ for all $x \in M$.

Theorem 7. If the data are analytic (that is, if $M$ is an analytic manifold and $F$ an analytic function) then $\mathfrak{C}_{x}=\mathfrak{H}_{x}$ for all $x \in M$.

Proof. We shall show that $\operatorname{dim} \mathfrak{C}_{x}$ is constant on $M$, so the result will follow from the corollary above. We proceed via a couple of lemmas.

Lemma 1. Take any $x \in M$ and any analytic curve $c(t)$ with $c(0)=x$. Let $V$ be any analytic section of $\mathfrak{H}$. For $|t|$ sufficiently small

$$
V_{c(t)}=\left.\rho_{c *}\right|_{0} ^{t}\left(\sum_{r=0}^{\infty} \frac{t^{r}}{r !}\left(\nabla_{\dot{c}}^{r} V\right)_{x}\right)
$$

where $\left.\rho_{c *}\right|_{0} ^{t}$ is the operator of parallel translation along $c$ from $c(0)=x$ to $c(t)$.

Proof. Let $\left\{v_{\alpha}(0)\right\}$ be a basis for $\mathfrak{H}_{x}$, and for each $\alpha$ let $v_{\alpha}(t)=\left.\rho_{c *}\right|_{0} ^{t} v_{\alpha}(0)$ be the element of $\mathfrak{H}_{c(t)}$ defined by parallel translation of $v_{\alpha}(0)$. Then $\left\{v_{\alpha}\right\}$ is a basis of sections of $\mathfrak{H}$ along $c$, and $\nabla_{\dot{c}} v_{\alpha}=0$ by construction. An analytic section $V$ of $\mathfrak{H}$, restricted to $c$, may be expressed in terms of $\left\{v_{\alpha}\right\}$ as

$$
V_{c(t)}=\nu^{\alpha}(t) v_{\alpha}(t)
$$

where the coefficients $\nu^{\alpha}$ are analytic functions of $t$. Thus

$$
\nu^{\alpha}(t)=\sum_{r=0}^{\infty} \frac{t^{r}}{r !} \frac{d^{r} \nu^{\alpha}}{d t^{r}}(0)
$$


for $|t|$ sufficiently small: to be precise, for $|t|$ less than the smallest of the radii of convergence of the functions $\nu^{\alpha}$ at 0 . For each $r=1,2, \ldots$,

$$
\left(\nabla_{\dot{c}}^{r} V\right)(t)=\frac{d^{r} \nu^{\alpha}}{d t^{r}}(t) v_{\alpha}(t)
$$

since $\nabla_{\dot{c}} v_{\alpha}=0$. Thus

$$
V_{c(t)}=\left.\left(\sum_{r=0}^{\infty} \frac{t^{r}}{r !} \frac{d^{r} \nu^{\alpha}}{d t^{r}}(0)\right) \rho_{c *}\right|_{0} ^{t} v_{\alpha}(0)=\left.\rho_{c *}\right|_{0} ^{t}\left(\sum_{r=0}^{\infty} \frac{t^{r}}{r !}\left(\nabla_{\dot{c}}^{r} V\right)_{x}\right),
$$

as required.

We wish to apply this formula with $V$ an element of $\mathfrak{C} \subset \operatorname{Sec}(\mathfrak{H})$. Now $\mathfrak{C}$ is spanned (over $C^{\omega}(M)$ ) by elements of the form

$$
\nabla_{X_{1}} \nabla_{X_{2}} \cdots \nabla_{X_{m}}(R(Y, Z))
$$

where $X_{1}, X_{2}, \ldots, X_{m}, Y$ and $Z$ are any vector fields on $M$. If we restrict our attention to a coordinate patch $U$ from the given analytic atlas then it will be enough to take $X_{1}, X_{2}, \ldots, X_{m}, Y$ and $Z$ to be coordinate fields. We take a local basis $\left\{V_{\alpha}\right\}$ of sections of $\mathfrak{H}$ over $U$ and set

$$
R\left(\frac{\partial}{\partial x^{j}}, \frac{\partial}{\partial x^{k}}\right)=R_{j k}=R_{j k}^{\alpha} V_{\alpha}
$$

the coefficients $R_{j k}^{\alpha}$ are functions on $U$, analytic in the coordinates. We abbreviate $\nabla_{\partial / \partial x^{i}}$ to $\nabla_{i} ;$ since $\nabla(\operatorname{Sec}(\mathfrak{H})) \subset \operatorname{Sec}(\mathfrak{H})$ we may set

$$
\nabla_{i} V_{\alpha}=\Gamma_{i \alpha}^{\beta} V_{\beta}
$$

the connection coefficients $\Gamma_{i \alpha}^{\beta}$ are functions on $U$, analytic in the coordinates. Thus for any $x \in U$ there is an open Euclidean coordinate ball $B_{\delta}(x)$ centred at $x$ such that the power series expansions about $x$, in terms of coordinates, of all the functions $R_{j k}^{\alpha}$ and $\Gamma_{i \alpha}^{\beta}$ converge in $B_{\delta}(x)$.

Lemma 2. The coefficients with respect to $\left\{V_{\alpha}\right\}$ of all sections of $\mathfrak{H}$ of the form $\nabla_{i_{1}} \nabla_{i_{2}} \cdots \nabla_{i_{m}} R_{j k}$ can be expanded in convergent power series about $x \in U$ in the same open coordinate ball $B_{\delta}(x)$.

Proof. If $f$ is an analytic function whose power series expansion about $x$ converges in $B_{\delta}(x)$, the power series expansion about $x$ of $\partial f / \partial x^{i}$ also converges in $B_{\delta}(x)$; and if $f_{1}$ and $f_{2}$ are analytic functions whose power series 
expansions about $x$ converge in $B_{\delta}(x)$, the power series expansion about $x$ of $f_{1} f_{2}$ also converges in $B_{\delta}(x)$. But

$$
\nabla_{i}\left(\nu^{\alpha} V_{\alpha}\right)=\left(\frac{\partial \nu^{\alpha}}{\partial x^{i}}+\Gamma_{i \beta}^{\alpha} \nu^{\beta}\right) V_{\alpha}
$$

Thus if the coefficients with respect to $\left\{V_{\alpha}\right\}$ of an analytic section $V$ of $\mathfrak{H}$ have power series expansions about $x$ that converge in $B_{\delta}(x)$ then the coefficients of $\nabla_{i} V$ have power series expansions about $x$ that also converge in $B_{\delta}(x)$. It follows that the coefficients of all sections of $\mathfrak{H}$ of the form $\nabla_{i_{1}} \nabla_{i_{2}} \cdots \nabla_{i_{m}} R_{j k}$ can be expanded in convergent power series about $x$ in $B_{\delta}(x)$.

We can now complete the proof of the theorem.

Let $U$ be a coordinate neighbourhood of $x$ in $M$ (from the analytic atlas) with $x$ as origin, whose image in $\mathbb{R}^{m}$ contains the ball $B_{\delta}(x)$. For any fixed $w \in T_{x} M$ whose coordinate representation has Euclidean length $\delta$ let $r_{w}$ be the ray starting at $x$ with initial tangent vector $w$, so that in coordinates $r_{w}(t)=\left(t w^{i}\right)$ with $\sum\left(w^{i}\right)^{2}=\delta^{2}$. Set $W=w^{i} \partial / \partial x^{i}$, so that $r_{w}$ is the integral curve of $W$ starting at $x$. By Lemma 2 the formula of Lemma 1 , which here becomes

$$
V_{r_{w}(t)}=\left.\rho_{r_{w} *}\right|_{0} ^{t}\left(\sum_{r=0}^{\infty} \frac{t^{r}}{r !}\left(\nabla_{W}^{r} V\right)_{x}\right),
$$

holds for all $t$ with $0 \leq t<1$, and for all $w$, where $V$ is any vector field of the form $\nabla_{i_{1}} \nabla_{i_{2}} \cdots \nabla_{i_{m}} R_{j k}$. The $V_{r_{w}(t)}$ span $\mathfrak{C}_{r_{w}(t)}$; each term in the sum belongs to $\mathfrak{C}_{x}$. That is to say, for each $t$ with $0 \leq t<1,\left.\mathfrak{C}_{r_{w}(t)} \subseteq \rho_{r_{w}}\right|_{0} ^{t}\left(\mathfrak{C}_{x}\right)$. Since parallel translation is an isomorphism, $\operatorname{dim} \mathfrak{C}_{r_{w}(t)} \leq \operatorname{dim} \mathfrak{C}_{x}$. There is thus a neighbourhood of $x$ in $M$ (namely $B_{\delta}(x)$ ) on which $\operatorname{dim} \mathfrak{C}_{y} \leq \operatorname{dim} \mathfrak{C}_{x}$. But we know that there is also a neighbourhood of $x$ in $M$ on which $\operatorname{dim} \mathfrak{C}_{y} \geq$ $\operatorname{dim} \mathfrak{C}_{x}$; so there is a neighbourhood of $x$ on which $\operatorname{dim} \mathfrak{C}_{y}=\operatorname{dim} \mathfrak{C}_{x}$. Thus $\operatorname{dim} \mathfrak{C}_{x}$ is locally constant on $M$, and so (assuming $M$ is connected) it is constant on $M$.

\section{Concluding remarks}

In this paper we have used techniques from the theory of Lie groupoids and Lie algebroids to investigate the infinitesimal holonomy of a certain class of fibre bundles whose the fibres are compact and support a fibre metric. We chose this particular case because it has an immediate application to 
the holonomy of the canonical horizontal lift or nonlinear connection of a Landsberg space in Finsler geometry, where the horizontal lifts of curves determine isometries between the fibres. We should, of course, like to generalise this approach, so as to be able to deal with Finsler spaces in general, and more generally with homogeneous nonlinear connections as in [6].

To do so we have to face a difficulty. One important role of the fibre metric in this paper was to ensure that the holonomy groupoid, in the case of a Landsberg space for example, is a subgroupoid of a Lie groupoid, namely the fibre-isometry groupoid. Without it, we have no obvious choice of an ambient Lie groupoid within which to locate the holonomy groupoid, even for Finsler spaces. Indeed, Muzsnay and Nagy [9] give an example of a Finsler space whose holonomy group is not a (finite-dimensional) Lie group. However, we can always work within the groupoid of fibre diffeomorphisms, though it will not have a finite-dimensional smooth structure. We expect, however, that it will still be possible to construct the equivalent of a Lie algebroid for this more general structure, and to represent sections of this 'algebroid' as projectable vector fields on the total space of the bundle. This expectation is based on material to be found in [8]; the fact that the fibres are actually (for Finsler spaces) or effectively (for homogenous nonlinear connections) compact is significant.

If this is indeed correct, then it seems to us that what remains of our account above when references to fibre metrics and fibre isometries are omitted should be a good guide to a theory of holonomy of Finsler spaces and homogeneous nonlinear connections. In particular, one can think of the horizontal lift as providing an 'infinitesimal connection' $\gamma$, with respect to which each projectable vector field can be written uniquely as the sum of a horizontal and a vertical vector feld. The vertical vector fields should then be thought of as sections of the 'kernel' of the 'algebroid'; that is, we now take seriously the suggestion made in Section 4 that the space of vertical vector fields on $T_{x}^{\circ} M$ should be regarded as the fibre of a 'vector bundle', indeed 'Lie algebra bundle', $\mathcal{V}$ over $M$. The covariant-derivative-like operator $\nabla$, which acts on $\operatorname{Sec}(\mathcal{V})$, and is related to the Berwald connection in the Finsler case or more generally to a so-called connection of Berwald type [3], will continue to play the role of $\nabla^{\gamma}$ for the 'infinitesimal connection'. The objects of interest are the 'Lie algebra sub-bundles' $\mathfrak{L}$ of $\mathcal{V}$ which contain all curvature vector fields (that is, such that for all $x \in M$ and all $v, w \in T_{x} M, R_{x}(v, w) \in \mathfrak{L}_{x}$ ), and satisfy $\nabla(\operatorname{Sec}(\mathfrak{L})) \subset \operatorname{Sec}(\mathfrak{L})$.

Let us for definiteness consider the Finsler case. For any $x \in M$ we may define the vector subspace $\mathfrak{R}_{x}$ of $\mathcal{V}_{x}$, the space of vertical vector fields on $\mathcal{I}_{x}$, much as before: it is the smallest subspace of $\mathcal{V}_{x}$ containing all fields 
$\rho_{c *} R_{y}(v, w)$ for any $y \in M$ and any curve $c$ in $M$ joining $y$ to $x$, and (now an extra condition) which is closed with respect to the compact $C^{\infty}$ topology (for details see [8]). Evidently for any curve $c$ joining $x$ and $y, \rho_{c *}: \mathfrak{R}_{x} \rightarrow \mathfrak{R}_{y}$ is an isomorphism. Set $\mathfrak{R}=\bigcup_{x} \mathfrak{R}_{x}$. Then by the ray construction used in the proof of Proposition $5, \mathfrak{R}$ is locally trivial, in the sense that $\left.\mathfrak{R}\right|_{U} \simeq$ $U \times \mathfrak{R}_{o}$ where $U$ is a suitable coordinate chart and $o$ its origin. We shall allow ourselves to think of $\Re$ as a vector bundle over $M$. We claim that $\nabla(\operatorname{Sec}(\mathfrak{R})) \subset \operatorname{Sec}(\mathfrak{R})$. Indeed, let $c$ be a curve in $M$ and $V$ a local section of $\mathfrak{R}$ over some $U$ containing $c(0)=x$, so that in particular $V(t)=V_{c(t)}$ depends smoothly on $t$ and $V(t) \in \mathfrak{R}_{c(t)}$. Now

$$
\nabla_{\dot{c}(0)} V=\frac{d}{d t}\left(\left(\left.\rho_{c *}\right|_{0} ^{t}\right)^{-1} V(t)\right)_{t=0},
$$

where $\left.\rho_{c *}\right|_{0} ^{t}: \mathfrak{R}_{x} \rightarrow \mathfrak{R}_{c(t)}$ is the isomorphism. Thus $\left(\left.\rho_{c *}\right|_{0} ^{t}\right)^{-1} V(t) \in \mathfrak{R}_{x}$ for all $t$, and so $\nabla_{\dot{c}(0)} V \in \mathfrak{R}_{x}$. That is, for any local section $V$ of $\mathfrak{R}$, for any $x$ in its domain and for any $v \in T_{x} M, \nabla_{v} V \in \mathfrak{R}_{x}$. We can now argue exactly as in the proof of Theorem 4 to conclude that $\mathfrak{R}$ is a Lie algebra sub-bundle of $\mathcal{V}$.

The drawback, of course, is that one cannot use the theory of Section 2 (which applies only to Lie groupoids) to argue that the holonomy Lie algebra bundle is the least Lie algebra sub-bundle of $\mathcal{V}$ which contains curvature fields and whose section space is invariant under $\nabla$. But it is certainly the case that $\mathfrak{R}$ satisfies these latter requirements, and $\mathfrak{R}_{x}$ is the obvious candidate for the role of holonomy algebra at $x$.

In [8], Michor also proposes that (in our notation) $\mathfrak{R}_{x}$ should be considered as the holonomy algebra at $x$; his analysis is different from ours, but leads nevertheless to the result that $\mathfrak{R}_{x}$ is a Lie algebra (his Lemma 12.3).

In a recent paper about holonomy of Finsler spaces [9], Muzsnay and Nagy express some reservations about this proposal of Michor's: they say, not unreasonably on the face of it, that 'the introduced holonomy algebras [i.e. the algebras $\mathfrak{R}_{x}$ ] could not be used to estimate the dimension of the holonomy group since their tangential properties to the holonomy group were not clarified'. It is true that Michor doesn't directly address the question of how one gets from the holonomy group at $x$ to $\mathfrak{R}_{x}$; his justification for calling $\mathfrak{R}_{x}$ the holonomy algebra at $x$ seems to be that when $\mathfrak{R}_{x}$ is finite-dimensional and generated by complete vector fields (as will be automatically the case when the fibre is compact) then it really is the holonomy algebra of a principal bundle with connection, as he shows. (We, of course, could argue in a somewhat similar vein, namely by pointing out that on specializing for example to the Landsberg case we obtain the correct answer.) 
But in fact Muzsnay and Nagy have the solution to their problem ready to hand, since a major part of Section 2 of their paper is devoted to discussing what it means for a vector field on $\mathcal{I}_{x}$ to be tangent to the holonomy group, and they show in Section 3 of their paper that the curvature fields $R_{x}(v, w)$ are tangent to the holonomy group at $x$ according to their definition. We shall show below, using their methods for the most part, that the elements of $\mathfrak{R}_{x}$ are indeed tangent to the holonomy group at $x$.

Before discussing this further, we wish to resolve a potential cause of confusion between their paper and ours. In [9] Muzsnay and Nagy introduce, for any Finsler space, the notion of the curvature algebra at $x \in M$, which they denote by $\mathfrak{R}_{x}$ : it is the Lie subalgebra of $\mathcal{V}_{x}$ 'generated by the curvature vector fields', i.e. the vector fields $R_{x}(v, w)$ as $v, w$ range over $T_{x} M$, for fixed $x$. We point out, for the sake of clarity, that their curvature algebra at $x$ is not the same as our $\mathfrak{R}_{x}$, and in general will be a proper subalgebra of it.

Now the restricted holonomy group at $x, H_{x}^{\circ}$, which corresponds to closed curves in $M$ which are contractible, is a subgroup of the diffeomorphism group $\operatorname{diff}\left(\mathcal{I}_{x}\right)$ of $\mathcal{I}_{x}$. Moreover, it has the property that every one of its elements can be connected to the identity diffeomorphism $\mathrm{id}_{\mathcal{I}_{x}}$ by a piecewise-smooth curve lying in $H_{x}^{\circ}$; just as in Section 2 this follows from the Factorization Lemma.

Let $\phi(t)$ be a curve in $\operatorname{diff}\left(\mathcal{I}_{x}\right)$, defined on some open interval $I$ containing 0 , such that $\phi(0)=\mathrm{id}_{\mathcal{I}_{x}}$, and which is differentiable enough for the following construction to make sense. For $u \in \mathcal{I}_{x}$ consider the curve $\phi_{u}$ in $\mathcal{I}_{x}$ defined by $\phi_{u}(t)=\phi(t) u$. Set

$$
V_{u}=\frac{d^{k}}{d t^{k}}\left(\phi_{u}\right)_{t=0}
$$

where $k \geq 1$ is the smallest integer for which the derivative is not zero for all $u$. Assume this defines a smooth vector field $V$ on $\mathcal{I}_{x}$. Muzsnay and Nagy show in effect that if we take curves $\phi(t)$ in the restricted holonomy group $H_{x}^{\circ}$ then the set of all such vector fields is an $\mathbb{R}$-linear space, closed under bracket; that is to say, it is a Lie algebra of vector fields. (It is necessary to allow $k>1$ in order to deal with, for example, the problem with the parametrization when defining the bracket in terms of the commutator of curves in the group.) But as we remarked above, every element of $H_{x}^{\circ}$ is connected to the identity by a curve in $H_{x}^{\circ}$, which makes this a pretty strong candidate to be called the holonomy algebra; we denote it by hol $(x)$ as in [9].

For any curve $c$ in $M$ joining $y$ to $x$, the map $H_{y}^{\circ} \rightarrow H_{x}^{\circ}$ defined by $\psi \mapsto \rho_{c} \circ \psi \circ \rho_{c}^{-1}$, where $\psi \in H_{y}^{\circ}$, is an isomorphism. Now let $\psi(t)$ be a curve in $H_{y}^{\circ}$ with $\psi(0)=\operatorname{id}_{\mathcal{I}_{y}}$; then $\phi: t \mapsto \rho_{c} \circ \psi(t) \circ \rho_{c}^{-1}$ is a curve in $H_{x}^{\circ}$ with 
$\phi(0)=\mathrm{id}_{\mathcal{I}_{x}}$. For $u \in \mathcal{I}_{x}$ we have

$$
\phi_{u}(t)=\rho_{c}\left(\psi_{\rho_{c}^{-1}(u)}(t)\right)
$$

so the element $V$ of $\operatorname{hol}(x)$ determined by $\phi$ is just $\rho_{c *} W$ where $W$ is the element of hol $(y)$ determined by $\psi$. It follows that $W \mapsto \rho_{c *} W$ is an isomorphism of Lie algebras $\operatorname{hol}(y) \rightarrow \operatorname{hol}(v)$. Now as Muzsnay and Nagy themselves show, for every $y \in M, \operatorname{hol}(y)$ contains the curvature fields $R_{y}(v, w)$. Thus hol $(x)$ must contain their parallel translates $\rho_{c *} R_{y}(v, w)$. It follows immediately that $\mathfrak{R}_{x} \subset \operatorname{hol}(x)$.

\section{Appendix 1: groupoids and algebroids}

In this section we recall the relevant definitions from the theory of Lie groupoids and Lie algebroids (more details may be found in Mackenzie's comprehensive work [7]).

Definition. A groupoid consists of two sets $\Omega$ and $M$, called respectively the groupoid and the base, together with two maps $\alpha$ and $\beta$ from $\Omega$ to $M$, called respectively the source projection and target projection, a map $1: x \mapsto$ $1_{x}, M \rightarrow \Omega$ called the object inclusion map, and a partial multiplication $(h, g) \mapsto h g$ in $\Omega$ defined on the set $\Omega * \Omega=\{(h, g) \in \Omega \times \Omega \mid \alpha(h)=\beta(g)\}$, all subject to the following conditions:

1. $\alpha(h g)=\alpha(g)$ and $\beta(h g)=\beta(h)$ for all $(h, g) \in \Omega * \Omega$;

2. $j(h g)=(j h) g$ for all $j, h, g \in \Omega$ such that $\alpha(j)=\beta(h)$ and $\alpha(h)=$ $\beta(g)$;

3. $\alpha\left(1_{x}\right)=\beta\left(1_{x}\right)=x$ for all $x \in M$;

4. $g 1_{\alpha(g)}=g$ and $1_{\beta(g)} g=g$ for all $g \in \Omega$;

5. each $g \in \Omega$ has a two-sided inverse $g^{-1}$ such that $\alpha\left(g^{-1}\right)=\beta(g)$, $\beta\left(g^{-1}\right)=\alpha(g)$ and $g^{-1} g=1_{\alpha(g)}, g g^{-1}=1_{\beta(g)}$.

We use the notation $r_{g}, l_{g}$ to denote right and left translation by $g \in \Omega$.

$A$ Lie groupoid is a groupoid $\Omega$ on base $M$ together with smooth structures on $\Omega$ and $M$ such that the maps $\alpha, \beta: \Omega \rightarrow M$ are surjective submersions, the object inclusion map $x \mapsto 1_{x}, M \rightarrow \Omega$ is smooth, and partial multiplication $\Omega * \Omega \rightarrow \Omega$ is smooth. We say that $\Omega$ is locally trivial if the map $(\alpha, \beta): \Omega \rightarrow M \times M$ is a surjective submersion. 
We adopt the convention that the elements of $\Omega * \Omega$ satisfy $\alpha(h)=\beta(g)$ as this is appropriate when the elements of the groupoid are maps whose arguments are on the right.

Definition. A Lie algebroid is a vector bundle $\pi: A \rightarrow M$ together with a Lie bracket on the (infinite-dimensional) vector space $\operatorname{Sec}(\pi)$ of sections of $\pi$ and a vector bundle map $a: A \rightarrow T M$ over the identity on $M$, the anchor map, which satisfies

$$
[\theta, f \phi]=f[\theta, \phi]+\left(\mathcal{L}_{a \circ \theta} f\right) \phi
$$

for any sections $\theta, \phi \in \operatorname{Sec}(\pi)$ and any function $f$ on $M$, and whose action on sections by composition is a Lie algebra homomorphism from $\operatorname{Sec}(\pi)$ to $\mathfrak{X}(M)$, the Lie algebra of vector fields on $M$.

We say that $A$ is transitive if the anchor map is surjective on each fibre.

The kernel $L$ of the anchor map $a$ is again a Lie algebroid, but of a rather special kind: its anchor map is by definition zero, and so the Lie bracket on sections of $L \rightarrow M$ induces a Lie bracket on each fibre of $L$. It may be shown that if $A$ is transitive then the fibres of $L$ are pairwise isomorphic as Lie algebras, so that $L$ is a Lie algebra bundle.

Every Lie groupoid $\Omega$ gives rise to an associated Lie algebroid $A \Omega$, in the following way. Put $A \Omega=\left.V \alpha\right|_{1(M)} \subset T \Omega$, the bundle of tangent vectors to $\Omega$ at points of the image of $1: M \rightarrow \Omega$ which are vertical with respect to the source projection $\alpha$; this is a vector bundle over $M$ in the obvious way. For any $g \in \Omega$ with $x=\alpha(g)$, right translation by $g$ induces a map $r_{g *}: T_{1_{x}} \Omega \rightarrow T_{g} \Omega$ which restricts to a map $V_{1_{x}} \alpha \rightarrow V_{g} \alpha$; in this way, any section of $A \Omega \rightarrow M$ may be extended by right translation to a vector field on $\Omega$ which is vertical over $\alpha$. The Lie bracket of any two such vector fields is again vertical over $\alpha$, and so we may define the Lie bracket of two sections to be the restriction, to $1(M)$, of the Lie bracket of the corresponding rightinvariant vector fields. Finally, the map $a=\left.\beta_{*}\right|_{A \Omega}$, the restriction of the tangent map to the target projection $\beta$, satisfies the conditions for an anchor map. We write $L \Omega$ for the kernel of the anchor map $a: A \Omega \rightarrow T M$.

If the base manifold $M$ is connected then the Lie groupoid $\Omega$ is locally trivial if, and only if, the associated Lie algebroid $A \Omega$ is transitive.

\section{Appendix 2: some general results about vector bun- dles with connections}

Proposition 11. Let $E \rightarrow M$ be a vector bundle equipped with a linear connection. Denote by $\nabla$ the corresponding covariant derivative, and for 
any curve $c$ in $M$ let $\tau_{c}: E_{x} \rightarrow E_{y}$ (where $c(0)=x, c(1)=y$ ) be the isomorphism given by parallel translation along $c$. For any vector sub-bundle $E^{\prime}$ of $E$, the following conditions are equivalent:

1. $\nabla\left(\operatorname{Sec}\left(E^{\prime}\right)\right) \subset \operatorname{Sec}\left(E^{\prime}\right)$; that is, for every section $\xi$ of $E^{\prime}$ and every vector field $X$ on $M, \nabla_{X} \xi$ is again a section of $E^{\prime}$;

2. for every curve $c, \tau_{c}$ restricts to an isomorphism of $E_{x}^{\prime}$ with $E_{y}^{\prime}$.

Proof. (1) $\Rightarrow(2)$ By the usual properties of connections we can define covariant derivatives of sections along curves. Let $\left\{\xi_{a}(t)\right\}$ be a local basis of sections of $E^{\prime}$ along $c(t)$ : then any local section of $E^{\prime}$ along $c$ can be written as a linear combination of the $\xi_{a}$ with coefficients which are functions of $t$. By assumption, $\nabla_{\dot{c}} \xi_{a}$ is a section of $E^{\prime}$; it may therefore be expressed as a linear combination of the $\xi_{a}$, say $\nabla_{\dot{c}} \xi_{a}=K_{a}^{b} \xi_{b}$ for some functions $K_{a}^{b}$ of $t$. We shall modify the $\xi_{a}$ so as to obtain a new local basis $\left\{\eta_{a}\right\}$ of sections of $E^{\prime}$ along $c$ such that $\nabla_{\dot{c}} \eta_{a}=0$. Consider the equations

$$
\frac{d}{d t}\left(\Lambda_{a}^{b}\right)+\Lambda_{a}^{c} K_{c}^{b}=0
$$

for functions $\Lambda_{a}^{b}$ of $t$. These are first-order linear ordinary differential equations for the unknowns $\Lambda_{a}^{b}$, and have a unique solution for initial conditions specified at $t=0$, which we may take to be $\Lambda_{a}^{b}(0)=\delta_{a}^{b}$ : then the solution, considered as a matrix, will be nonsingular at all points of $c$ sufficiently close to $x$. Without loss of generality we may assume that $y$ lies within the neighbourhood on which $\left(\Lambda_{a}^{b}\right)$ is nonsingular. Then if $\eta_{a}(t)=\Lambda_{a}^{b}(t) \xi_{b}(t)$, $\left\{\eta_{a}\right\}$ is a new basis of sections of $E^{\prime}$ along $c$ such that

$$
\nabla_{\dot{c}} \eta_{a}=\Lambda_{a}^{b} \nabla_{\dot{c}} \xi_{b}+\frac{d}{d t}\left(\Lambda_{a}^{b}\right) \xi_{b}=0 .
$$

But this is just the condition for $\eta_{a}$ to be parallel along $c$, that is, for $\tau_{c} \eta_{a}(0)=\eta_{a}(1)$. So there are bases of $E_{x}^{\prime}$ and $E_{y}^{\prime}$ with respect to which $\tau_{c}$ is represented by the identity matrix.

$(2) \Rightarrow(1) \quad$ Let $c$ be smooth a curve in $M$ and $\eta$ a local section of $E^{\prime}$ over some neighbourhood of $c(0)=x$, so that $\eta(t)=\eta_{c(t)}$ depends smoothly on $t$ and $\eta(t) \in E_{c(t)}^{\prime}$. Now

$$
\nabla_{\dot{c}(0)} V=\frac{d}{d t}\left(\left(\left.\tau_{c}\right|_{0} ^{t}\right)^{-1} \eta(t)\right)_{t=0},
$$

where $\left.\tau_{c}\right|_{0} ^{t}: E_{x}^{\prime} \rightarrow E_{c(t)}^{\prime}$ is the isomorphism determined by parallel transport. Thus $\left(\left.\tau_{c}\right|_{0} ^{t}\right)^{-1} \eta(t) \in E_{x}^{\prime}$ for all $t$, and so $\nabla_{\dot{c}(0)} \eta \in E_{x}^{\prime}$. 
Proposition 12. Let $\pi: E \rightarrow M$ be a vector bundle equipped with a linear connection $\nabla$. Let $E^{\prime}$ be a subset of $E$ such that

1. $\pi$ maps $E^{\prime}$ onto $M$;

2. for each $x \in M, E_{x}^{\prime}=\left.\pi\right|_{E^{\prime}} ^{-1}(x)$ is a linear subspace of $E_{x}$;

3. for any pair of points $x, y \in M$ and any curve $c$ in $M$ joining them, $\tau_{c}\left(E_{x}^{\prime}\right) \subseteq E_{y}^{\prime}$.

Then $E^{\prime}$ is a vector sub-bundle of $E$ and $\nabla\left(\operatorname{Sec}\left(E^{\prime}\right)\right) \subset \operatorname{Sec}\left(E^{\prime}\right)$.

Proof. For any curve $c, \tau_{c}: E_{x} \rightarrow E_{y}$ has an inverse, namely $\tau_{\bar{c}}$, where $\bar{c}$ is the curve with the same image as $c$ but followed in the opposite direction: $\bar{c}(t)=c(1-t)$. Thus any point $e \in E_{y}^{\prime}$ is the image under $\tau_{c}$ of a point of $E_{x}^{\prime}$, namely $\tau_{\bar{c}}(e)$; and so $\tau_{c}: E_{x}^{\prime} \rightarrow E_{y}^{\prime}$ is both injective and surjective and so an isomorphism.

We use the ray construction, as in the proof of Proposition 5. Fix a point $o \in M$ and take a coordinate neighbourhood $U$ of $o$ with $o$ as origin such that the image of $U$ is the open unit ball in $\mathbb{R}^{m}$. For each $x \in U$ let $r_{x}$ be the ray joining $o$ to $x$ with respect to the coordinates. We further assume that $U$ is contained in some neighbourhood over which $E$ is locally trivial; then in terms of the coordinates on $M$ and the corresponding fibre coordinates on $E$, for any $e \in E_{o}, \tau_{r_{x}} e$ is the solution of a system of ordinary differential equations in which the coordinates of $x$ play the role of parameters. The solutions of such equations depend smoothly on parameters, so if we set $\xi(x)=\tau_{r_{x}} e$ then $\xi$ is a smooth local section of $E$. Now choose a basis $\left\{e_{a}\right\}$ for $E_{o}^{\prime}$, and for each $x \in U$ set $\xi_{a}(x)=\tau_{r_{x}} e_{a}$. Then $\left\{\xi_{a}(x)\right\}$ is a basis of $E_{x}^{\prime}$, and $\left\{\xi_{a}\right\}$ is a basis of smooth local sections of $E^{\prime}$.

This construction provides at the same time local fibre coordinates on $E^{\prime}$ and bases of local sections of it. Let $U$ be one coordinate neighbourhood in $M$, with origin $o$, for such a system of local sections, and $V$, with origin $p$, another. We choose a basis $\left\{e_{a}\right\}$ for $E_{o}^{\prime}$ and a basis $\left\{f_{a}\right\}$ of $E_{p}^{\prime}$ to begin the construction in each case. We may assume that $\left\{f_{a}\right\}$ is the parallel translate of $\left\{e_{a}\right\}$ along some curve $c$ joining $o$ and $p$ (to assume otherwise merely involves interposing an additional constant non-singular coefficient matrix in the expression for transition functions shortly to be derived). We denote by $\left\{\xi_{a}\right\}$ the basis of local sections of $E^{\prime}$ determined by $o, U$ and $\left\{e_{a}\right\}$, and $\left\{\eta_{a}\right\}$ the basis determined by $p,\left\{f_{a}\right\}$ and $V$. Then for any $x \in$ $U \cap V, \eta_{a}(x)=\tau_{V, x} \tau_{c} \tau_{U, x}^{-1} \xi_{a}(x)$, where we have indexed the parallel transport operators along rays by the relevant coordinate patch. But $\tau_{V, x} \tau_{c} \tau_{U, x}^{-1}$ is an 
isomorphism of $E_{x}^{\prime}$, whose matrix with respect to the basis $\left\{\xi_{a}(x)\right\}$ depends smoothly on $x \in U \cap V$. This shows that $E^{\prime}$ is a smooth vector sub-bundle of $E$. By Proposition $11, \nabla\left(\operatorname{Sec}\left(E^{\prime}\right)\right) \subset \operatorname{Sec}\left(E^{\prime}\right)$.

Corollary 3. Let $E \rightarrow M$ be a vector bundle equipped with a linear connection, and let $E^{1}$ and $E^{2}$ be vector sub-bundles of $E$ such that $\nabla\left(\operatorname{Sec}\left(E^{i}\right)\right) \subset$ $\operatorname{Sec}\left(E^{)}, i=1,2\right.$. Then $E^{1} \cap E^{2}$ is a vector bundle.

Proof. By Proposition 11, parallel translation preserves both $E^{1}$ and $E^{2}$, so preserves their intersection. The result now follows from Proposition 12.

Proposition 13. If $E \rightarrow M$ is a Lie algebra bundle and $\nabla$ a connection on $E$ then $\nabla$ is a Lie connection if and only if for every curve $c$ in $M$, $\tau_{c}: E_{x} \rightarrow E_{y}$ is an isomorphism of Lie algebras.

Proof. Suppose that $\tau_{c}$ is always a Lie algebra isomorphism. Take a basis $\left\{e_{a}\right\}$ for the Lie algebra $E_{x}$, and set $e_{a}(t)=\left.\tau_{c}\right|_{0} ^{t} e_{a}$, where $\left.\tau_{c}\right|_{0} ^{t}$ is parallel translation along $c$ from $x=c(0)$ to $c(t)$. Then $\left\{e_{a}(t)\right\}$ is a basis for $E_{c(t)}$, and $\left[e_{a}(t), e_{b}(t)\right]=\left.\tau_{c}\right|_{0} ^{t}\left[e_{a}, e_{b}\right]$. So if we set $\left[e_{a}, e_{b}\right]=C_{a b}^{c} e_{c}$ then $\left[e_{a}(t), e_{b}(t)\right]=C_{a b}^{c} e_{c}(t)$. Now $\nabla_{\dot{c}} e_{a}(t)=0$, and so for any section $\xi(t)$ of $E$ along $c$, say $\xi(t)=\xi^{a}(t) e_{a}(t)$, we have $\nabla_{\dot{c}} \xi=\dot{\xi}^{a} e_{a}$. But for sections $\xi, \eta$ along $c$ we have $[\xi, \eta]=\xi^{a} \eta^{b} C_{a b}^{c} e_{c}$, whence

$$
\nabla_{\dot{c}}[\xi, \eta]=\frac{d}{d t}\left(\xi^{a} \eta^{b}\right) C_{a b}^{c} e_{c}=\left(\dot{\xi} \eta^{b}+\xi^{a} \dot{\eta}^{b}\right) C_{a b}^{c} e_{c}=\left[\nabla_{\dot{c}} \xi, \eta\right]+\left[\xi, \nabla_{\dot{c}} \eta\right]
$$

Conversely, suppose that $\nabla$ is Lie. Let $\left\{e_{a}(t)\right\}$ be a basis of parallel sections of $E$ along $c(t)$, so that $\nabla_{\dot{c}} e_{a}=0$. Then if we set $\left[e_{a}(t), e_{b}(t)\right]=C_{a b}^{c}(t) e_{c}(t)$, it is evident from the Lie property that the $C_{a b}^{c}$ are actually constants. But $e_{a}(t)=\left.\tau_{c}\right|_{0} ^{t} e_{a}(0)$, so

$$
\begin{aligned}
{\left[\left.\tau_{c}\right|_{0} ^{t} e_{a}(0),\left.\tau_{c}\right|_{0} ^{t} e_{b}(0)\right] } & =\left[e_{a}(t), e_{b}(t)\right] \\
& =C_{a b}^{c}(t) e_{c}(t)=\left.C_{a b}^{c}(0) \tau_{c}\right|_{0} ^{t} e_{c}(0)=\left.\tau_{c}\right|_{0} ^{t}\left[e_{a}(0), e_{b}(0)\right],
\end{aligned}
$$

which says that $\left.\tau_{c}\right|_{0} ^{t}$ is a Lie algebra isomorphism.

Corollary 4. Let $E \rightarrow M$ be a Lie algebra bundle equipped with a Lie connection, and let $E^{1}$ and $E^{2}$ be Lie algebra sub-bundles of $E$ such that $\nabla\left(\operatorname{Sec}\left(E^{i}\right)\right) \subset \operatorname{Sec}\left(E^{i}\right), i=1,2$. Then $E^{1} \cap E^{2}$ is a Lie algebra bundle.

Proof. By Corollary $3, E^{1} \cap E^{2}$ is a vector bundle. Each fibre is a Lie algebra, and by Proposition 13 the fibres are pairwise isomorphic as Lie algebras. 


\section{Acknowledgements}

The first author is a Guest Professor at Ghent University: he is grateful to the Department of Mathematics for its hospitality. The second author acknowledges the support of grant no. 201/09/0981 for Global Analysis and its Applications from the Czech Science Foundation.

\section{Address for correspondence}

M. Crampin, 65 Mount Pleasant, Aspley Guise, Beds MK17 8JX, UK

Crampin@btinternet.com

\section{References}

[1] D. Bao: On two curvature driven problems in Riemann-Finsler geometry Adv. Stud. Pure Math. 48 (2007) 19-71

[2] D. Bao, S.-S. Chern and Z. Shen: An Introduction to Riemann-Finsler Geometry (Springer, 2000)

[3] M. Crampin: Connections of Berwald type Publ. Math. Debrecen 57 (2000) 455-473

[4] M. Crampin: On Landsberg spaces and the Landsberg-Berwald problem Houston J. Math. to appear

[5] S. Kobayashi and K. Nomizu: Foundations of Differential Geometry Vol. I (Interscience, 1963)

[6] L. Kozma: Holonomy structures in Finsler geometry, in Handbook of Finsler Geometry ed. Antonelli (Kluwer, 2003)

[7] K. C.H. Mackenzie: General Theory of Lie Groupoids and Lie Algebroids (Cambridge University Press, 2005)

[8] P. W. Michor: Gauge Theory for Fibre Bundles (Bibliopolis, Napoli, 1991)

[9] Z. Muzsnay and P. T. Nagy: Finsler manifolds with non-Riemannian holonomy Houston J. Math. to appear

[10] Z. Shen: Differential Geometry of Spray and Finsler Spaces (Kluwer, 2001) 